\title{
Deficits in Enrichment-Dependent Neurogenesis and Enhanced Anxiety Behaviors Mediated by Expression of Alzheimer's Disease-Linked Ps1 Variants Are Rescued by Microglial Depletion
}

\author{
Sylvia Ortega-Martinez, ${ }^{1}$ Nisha Palla, ${ }^{2}$ Xiaoqiong Zhang, ${ }^{1}$ Erin Lipman, ${ }^{3}$ and Sangram S. Sisodia ${ }^{1}$ \\ Departments of ${ }^{1}$ Neurobiology, ${ }^{2}$ B.A. Neuroscience, and ${ }^{3}$ Statistics, The University of Chicago, Chicago, Illinois 60637
}

\begin{abstract}
Alzheimer's disease $(\mathrm{AD})$ is a progressive neurodegenerative disorder that presently affects an estimated 5.7 million Americans. Understanding the basis for this disease is key for the development of a future successful treatment. In this effort, we previously reported that mouse prion protein-promoter-driven, ubiquitous expression of familial AD (FAD)-linked human PSEN1 variants in transgenic mice impairs environmental enrichment (EE)-induced proliferation and neurogenesis of adult hippocampal neural progenitor cells (AHN$\mathrm{PCs}$ ) and in a non-cell autonomous manner. These findings were confirmed in $P S 1^{M 146 V /+}$ mice that harbor an FAD-linked mutation in the endogenous PSEN1 gene. We now demonstrate that CSF1R antagonist-mediated microglial depletion in transgenic male mice expressing mutant presenilin 1 (PS1) or $P S 1^{M 146 V /+}$ "knock-in" mice leads to a complete rescue of deficits in proliferation, differentiation and survival of AHNPCs. Moreover, microglia depletion suppressed the heightened baseline anxiety behavior observed in transgenic mice expressing mutant PS1 and $P S 1^{M 146 V /+}$ mice to levels observed in mice expressing wild-type human PS1 or nontransgenic mice, respectively. These findings demonstrate that in mice expressing FAD-linked PS1, microglia play a critical role in the regulation of EE-dependent AHNPC proliferation and neurogenesis and the modulation of affective behaviors.
\end{abstract}

Key words: Alzheimer; anxiety behaviors; environmental enrichment; hippocampal neurogenesis; microglia; presenilin

\section{Significance Statement}

Inheritance of mutations in genes encoding presenilin 1 (PS1) causes familial Alzheimer's disease (FAD). Mutant PS1 expression enhances the levels and assembly of toxic $A \beta 42$ peptides and impairs the self-renewal and neuronal differentiation of adult hippocampal neural progenitor cells (AHNPCs) following environmental enrichment (EE) that is associated with heightened baseline anxiety. We now show that microglial depletion fully restores the EE-mediated impairments in AHNPC phenotypes and suppresses the heightened baseline anxiety observed in mice expressing FAD-linked PS1. Thus, we conclude that the memory deficits and anxiety-related behaviors in patients with PS1 mutations is a reflection not just of an increase in the levels of A $\beta 42$ peptides, but to impairments in the self-renewal and neuronal differentiation of AHNPCs that modulate affective behaviors.

\section{Introduction}

Alzheimer's disease (AD), a progressive neurodegenerative disorder, is characterized by the presence of extracellular deposits of

Received April 18, 2019; revised May 13, 2019; accepted June 8, 2019.

Author contributions: S.0.-M. and S.S.S. designed research; S.0.-M., N.P., X.Z., and E.L. performed research; S.0.-M. analyzed data; S.0.-M. and S.S.S. wrote the paper.

This work was supported by NIH AG027854 and the Edward H. Levi Fund. S.S.S. is a paid Consultant of AZTherapies. We thank Jonathan Foldi and Keshav Vemuri for useful comments editing the paper, and help with other statistics analysis (PCA) that are not included in the final paper; Dr. Myles Minter and Pablo Oyler-Castrillo for earlier pilot experiments; Shirley Bond, Dr. Christine Labno, and Dr. Vytas Bindokas from the UChicago Light Microscopy Core for expert technical assistance with confocal microscopy; and Dr. Vega Asensio (Norarte Studio) and Jessica Xia for their help with the illustrations for the graphical abstract.

The authors declare no competing financial interests.

Correspondence should be addressed to Sangram S. Sisodia at ssisodia@bsd.uchicago.edu. $\beta$-amyloid $(\mathrm{A} \beta$ ) peptides in senile plaques, intraneuronal inclusions composed of hyperphosphorylated tau in neurofibrillary tangles, synaptic dysfunction, neuronal loss, and deterioration of cognitive function (Bloom, 2014).

The histopathological alterations in the brains of patients with $\mathrm{AD}$ have been associated with deficits in the functioning of the hippocampus, a region that plays a critical role in learning and memory (Squire, 1992). It has been well established that AHNPCs that reside in the subgranular zone (SGZ) of the dentate gyrus (DG) give rise to mature granule cells that integrate into

https://doi.org/10.1523/JNEUROSCI.0884-19.2019

Copyright $(2019$ the authors 
functional brain networks that are essential for hippocampal plasticity (Ortega-Martínez, 2015). Increased hippocampal neurogenesis in adulthood has been linked to improved hippocampal functioning that includes emotional functional networks, learning, and memory consolidation (Deng et al., 2010; Christian et al., 2014; Hill et al., 2015). Furthermore, exposure to an enriched environment (EE) increases AHNPC proliferation and increases the levels of granule cells in the DG (Matsumori et al., 2006).

Early onset, autosomal dominant forms of familial AD (FAD) are caused by expression of mutant genes encoding presenilins (PS1 and PS2), and amyloid precursor protein (APP) variants (Price and Sisodia, 1998). Presenilins are the catalytic components of $\mathrm{y}$-secretase, an enzyme complex that catalyzes intramembranous processing of $>100$ type 1 membrane proteins, including APP and Notch-1 (De Strooper, 2003) and PS1 has been shown to play a regulatory role in promoting adult hippocampal neurogenesis (AHN; Gadadhar et al., 2011; Bonds et al., 2015). Finally, we have documented that in transgenic expressing FAD-linked huPS1 $\mathrm{E} 9$ and huPS1M146L variants or $P S 1^{M 146 V /+}$ "knock-in" mice significantly impairs EE-mediated proliferation of AHNPCs and neurogenesis, phenotypes that are driven by non-cell-autonomous mechanism(s) (Choi et al., 2008; Veeraraghavalu et al., 2013).

To ascertain the role of cell types in the hippocampal niche that regulate neurogenesis in the context of $\mathrm{AD}$, there has been an increasing interest in the role of microglia (Weitz and Town, 2012; Cai et al., 2014). The microglial activities thought to contribute to $\mathrm{AD}$ pathogenesis include secretion of proinflammatory innate cytokines (Meda et al., 1995), but microglia also play important roles in clearance of cellular debris and homeostasis within the hippocampus (Neumann et al., 2009; Ravichandran, 2003). In this regard, we have demonstrated that chemokines and growth factors secreted from microglia expressing FAD-linked mutant PS1 significantly impairs proliferation and neuronal differentiation of neurospheres from mice expressing wild-type PS1 (Choi et al., 2008). To assess the role of microglia in mediating the deficits in EE-mediated AHNPC proliferation and differentiation observed in mice expressing FAD-linked mutant PS1 variants, we depleted microglia using a selective CSF1R kinase inhibitor, PLX5622. Our studies now reveal that that microglial depletion fully restores EE-mediated deficits in AHNPC proliferation and differentiation in transgenic mice expressing FAD-linked PS1 or $\mathrm{PS1}^{\mathrm{M146V/+}}$ mice to the levels observed in mice expressing their wild-type counterparts.

Clinically, although cognitive impairment is a principal feature of $\mathrm{AD}$, emotional dysfunction in the form of anxiety is one of the most common symptoms in patients with both early- and late-onset forms of $\mathrm{AD}$ (Ferreira et al., 2018). It is established that AHN is tightly linked to both cognitive and emotional networks and that impairments in AHN correlates with an increase in anxiety behaviors; promoting AHN is sufficient to decrease anxiety behaviors (Revest et al., 2009; Hill et al., 2015; Mohammad et al., 2018) In this regard, we observed heightened baseline anxiety in transgenic mice expressing the FAD-linked variants or $P S 1^{M 146 V /+}$ mice that were housed in either standard housing $(\mathrm{SH})$ or $\mathrm{EE}$ conditions compared with their wild-type counterparts. Remarkably, PLX5622-mediated microglial depletion fully suppressed baseline anxiety in mice expressing the PS1 variants to levels observed in their wild-type counterparts. Collectively, our studies document that the microglial depletion restores the impairments in EE-mediated AHNPC proliferation, survival and differentia- tion and suppresses heightened anxiety-like behaviors in mice expressing FAD-linked PS1.

\section{Materials and Methods}

\section{Animals}

Male mice expressing murine prion promoter-driven PS1 transgenes hPS1WT (line S8-4), PS1 EE9 (line S9), PS1M146L (line S15; Feng et al., 2001; Veeraraghavalu and Sisodia, 2013) were maintained in a (C3H/ $\mathrm{HeJ} \times \mathrm{C} 57 \mathrm{BL} / 6 \mathrm{~J} \mathrm{~F} 3) \times \mathrm{C} 57 \mathrm{BL} / 6 \mathrm{~J} \mathrm{n} 1$ background (Thinakaran et al.,

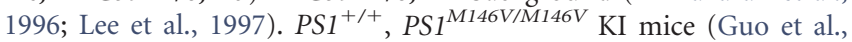
$1999)$, were maintained in a $(\mathrm{C} 3 \mathrm{H} / \mathrm{HeJ} \times \mathrm{C} 57 \mathrm{BL} / 6 \mathrm{~J} \mathrm{~F} 3) \times \mathrm{C} 57 \mathrm{BL} / 6 \mathrm{~J} \mathrm{n} 1$ background. $P S 1^{+/+}$were crossed to $P S 1^{M 146 V / M 146 V}$ KI mice to obtain heterozygous $P S 1^{M 146 V /+}$ mice.

We chose male mice for the analysis for two reasons: first, our earlier studies of the role of mutant presenilins in regulating hippocampal neurogenesis (Choi et al., 2008; Veeraraghavalu and Sisodia, 2013) was performed solely with male mice. The rationale was that the vast majority of environmental enrichment studies in the literature have been performed with male mice to avoid confounding issues pertaining to hormonal changes in females that are known to interfere with behavioral outputs. Second, it is well established that group-housed females are prone to develop dominance hierarchies that are extremely stressful that might impact the behavioral and physiological outcomes reported herein. However, it remains plausible that a sex difference might exist in the neurogenesis phenotype, or in the response to microglial depletion.

Animal experiments were conducted in accordance with institutional and National Institute of Health guidelines.

All murine experimental procedures were approved by the Institutional Animal Care and Use Committee at the University of Chicago and performed in accordance with approved Animal Care and Use Protocols.

\section{Experimental design}

The experimental design performed with each group of animals is described in Figure 1, $A$ and $B$. The total number of mice used per experiment is described in Tables 1-3.

\section{Environmental enrichment setting}

Using a similar protocol previously described for exposure to EE (Sztainberg and Chen, 2010), cohorts of the aforementioned 5-week-old male transgenic animals were housed in large cages containing running wheels, tunnels, toys, and chewable materials for 1 month. Control groups of animals were maintained in standard laboratory housing conditions.

\section{Compounds}

PLX5622 was provided by Plexxikon and formulated in AIN-76A standard chow by Research Diets at the 1200 ppm dose previously used (Dagher et al., 2015). The efficacy of the diet was proof in all mice by Ibal staining; checking all mice under PLX5622 presented a 99\% of microglia depletion in DG.

\section{Thymidine analogs injections}

BrdU injections: proliferation and survival studies in transgenic mice. hPS1WT (line S8-4), PS1 $\Delta$ E9 (line S9), PS1M146L (line S15) mice received a single intraperitoneal injection of BrdU (100 mg/kg; SigmaAldrich). In the proliferation studies, the BrdU injection was done on the last day of the experiment (standard housing or EE). Twenty-four hours later, mice were killed. In the survival studies, mice received a single intraperitoneal injection of BrdU after $15 \mathrm{~d}$ of EE or SH conditions and the mice continued under standard or EE conditions for 2 more weeks, when the mice were then killed and processed to determine survival and neuronal differentiation of the newborn cells.

CldU and IdU injections: proliferation and survival studies in nontransgenic and KI mice. $P S 1^{+/+}, P S 1^{M 146 V /+}$ KI mice were injected with 5-chloro-2' -deoxyuridine (CldU; $57.65 \mathrm{mg} / \mathrm{kg}$ body weight, i.p.; SigmaAldrich) dissolved in saline buffer $(0.9 \%)$ after $15 \mathrm{~d}$ of EE or SH conditions. After 2 weeks, mice then received another injection of 5-iodo-2'-deoxy-uridine (IdU; $42.75 \mathrm{mg} / \mathrm{kg}$ body weight, i.p.; SigmaAldrich) dissolved in PBS, as described previously (Ortega-Martínez and Trejo, 2015). One day after IdU injection, the animals were killed by 

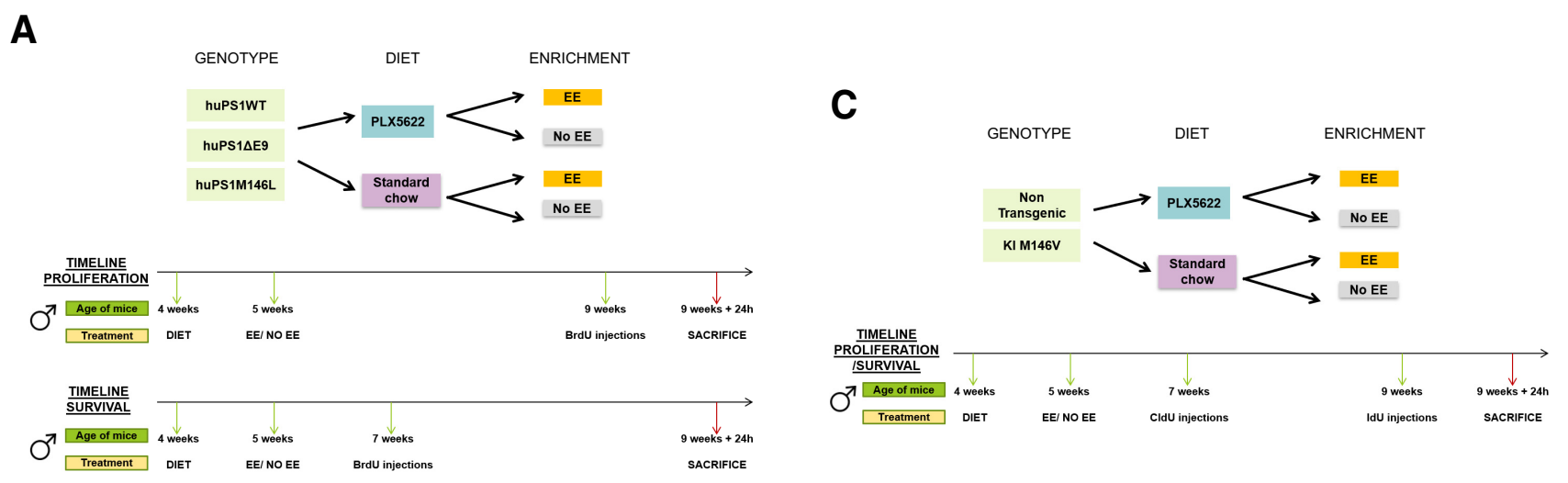

B

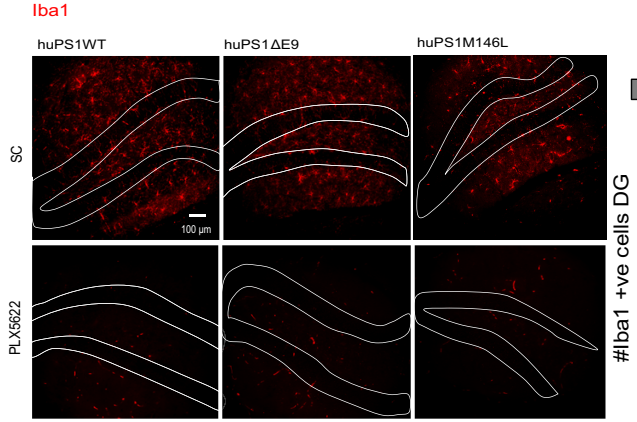

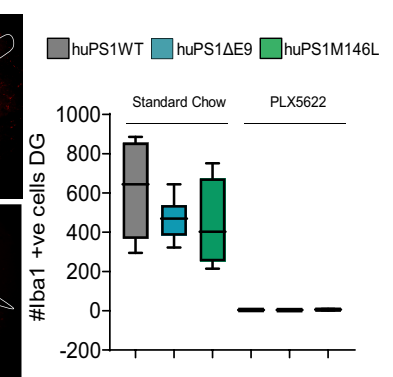

D

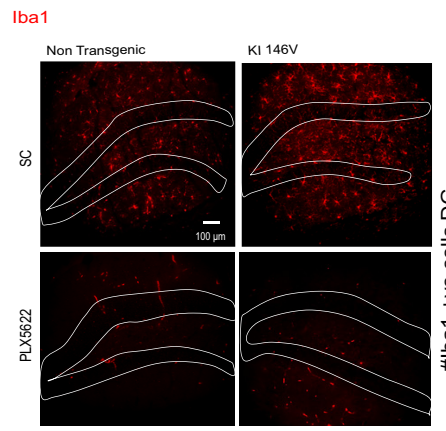

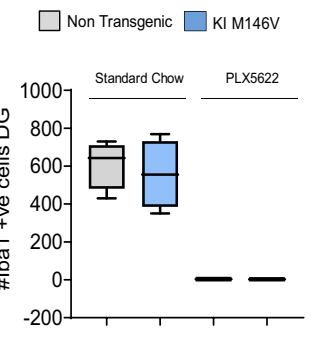

Figure 1. Experimental design. $\boldsymbol{A}$, PrP promoter-driven transgenic mice. Details of the genotype, diet, and EE cages. Experiments were started when mice were 1 month of age. $\boldsymbol{B}$, Images of Iba1-positive cells in hippocampi from each transgenic line maintained in SC and PLX5622 diets. Images were acquired with a Leica Sp8 confocal microscope under $20 \times$ objective. Scale bar, $50 \mu \mathrm{m}$. Quantifications of Iba1 + cells in DG of all transgenic line maintained in SC and PLX5622 diets. PLX5522 diet leads to a 99\% of microglia depletion. C, Nontransgenic and PS1 ${ }^{\mathrm{M} 146 \mathrm{~V} /+}$ mice. Details of the genotype, diet, and EE cages used. Nontransgenic and PS1 $1^{\mathrm{M} 146 \mathrm{~V} /+}$ mice were injected at 7 weeks with CldU for survival analysis. Experiments were started when mice were 1 month of age. D, Images of Iba1-positive cells in nontransgenic and PS1 ${ }^{\mathrm{M146V} /+} \mathrm{KI}$. Images were acquired with a Leica Sp8 confocal microscope under $20 \times$ objective. Scale bar, $50 \mu \mathrm{m}$. Quantification of Iba1+ cells in DG of nontransgenic and PS1 ${ }^{\mathrm{M} 146 \mathrm{~V} /+} \mathrm{KI}$ mice showing a 99\% of microglia depletion under PLX5622 diet.

Table 1. Detailed mice number used in the proliferation study

\begin{tabular}{lc}
\hline Groups of the proliferation study & No. of mice used, $n$ \\
\hline huPS1WT/SC/N0 EE & 8 \\
huPS1 $\Delta$ E9/SC/N0 EE & 7 \\
PS1M146L/SC/NO EE & 7 \\
huPS1WT/SC/EE & 6 \\
huPS1 $E$ E/SC/EE & 7 \\
PS1M146L/SC/EE & 9 \\
huPS1WT/PLX/N0 EE & 7 \\
huPS1 EE9/PLX/N0 EE & 6 \\
PS1M146L/PLX/N0 EE & 7 \\
huPS1WT/PLX/EE & 6 \\
huPS1 EE/PLX/EE & 7 \\
PS1M146L/PLX/EE & 12 \\
Total mice used & 89 \\
\hline
\end{tabular}

intracardiac perfusion. The doses of the thymidine analogs were based on equimolar doses of $100 \mathrm{mg} / \mathrm{kg}$ body weight BrdU. CldU was used to analyze cell survival. IdU injection was used to assess proliferation.

Perfusion and tissue processing

Mice were terminally killed by $\mathrm{CO}_{2}$ gas and underwent intracardial perfusion with cold $9 \% \mathrm{NaCl}$. Brains were removed and postfixed in paraformaldehyde (4\%) for $48 \mathrm{~h}$ at room temperature. Coronal sections (40 $\mu \mathrm{m})$ were cut and collected in PBS using a vibratome (Leica, VT1000S). Stereological series from the right hemisphere of the hippocampal formation were established using 96-well plates, with every sixth section for
Table 2. Detailed mice number used in the survival study

\begin{tabular}{lc}
\hline Groups of the survival study & No. of mice used, $n$ \\
\hline huPS1WT/SC/NO EE & 8 \\
huPS1 $\Delta$ E9/SC/N0 EE & 8 \\
PS1M146L/SC/NO EE & 6 \\
huPS1WT/SC/EE & 9 \\
huPS1 $E$ E9/SC/EE & 6 \\
PS1M146L/SC/EE & 8 \\
huPS1WT/PLX/N0 EE & 11 \\
huPS1 E E/PLX/N0 EE & 5 \\
PS1M146L/PLX/N0 EE & 6 \\
huPS1WT/PLX/EE & 9 \\
huPS1 $\triangle$ E9/PLX/EE & 5 \\
PS1M146L/PLX/EE & 9 \\
Total mice used & 90
\end{tabular}

immunohistochemistry analysis, as described previously (OrtegaMartínez and Trejo, 2015; Mohammad et al., 2018).

Briefly, free-floating sections were postfixed in 4\% PFA for 15 min and immersed in 50\% ethanol in PBS to improve antibody penetration. Slices were permeabilized with Triton X-100 (1\%) in PBS for $10 \mathrm{~min}$. For BrdU, $\mathrm{CldU}$, and IdU detection, antigen retrieval was done by incubation with formamide/SSC solution at $65^{\circ} \mathrm{C}$ for $2 \mathrm{~h}$ in a water bath. Slices were blocked using $10 \%$ donkey serum or $1 \%$ BSA in PBS containing $0.4 \%$ Triton X-100 for $2 \mathrm{~h}$.

Double, triple, or quadruple immunohistochemistry was performed using free-floating sections, incubating the following primary antibodies 
Table 3. Detailed mice number used in the nontransgenic/KI study

\begin{tabular}{lc}
\hline Groups nontransgenic/KI study & No. of mice used, $n$ \\
\hline Nontransgenic/SC/NO EE & 9 \\
KI M146V/SC/NO EE & 7 \\
Non Transgenic/SC/EE & 10 \\
KI M146V/SC/EE & 9 \\
Non Transgenic/PLX/NO EE & 7 \\
KI M146V/PLX/NO EE & 7 \\
Nontransgenic/PLX/EE & 8 \\
KI M146V/PLX/EE & 10 \\
Total mice used & 67
\end{tabular}

for dilutions were used, by $24-48 \mathrm{~h}$ incubations at $4^{\circ} \mathrm{C}$ : $1: 300$ rat antiBrdU (Abcam), 1:100 rat anti-CldU (Accurate Chemicals); 1:500 mouse anti-IdU (BD Biosciences);1:500 goat anti-doublecortin (Santa Cruz Biotechnology); 1:500 rabbit anti-MCM2 (Abcam); 1:500 mouse antiglial fibrillary acidic protein (GFAP) clone G-A-5 (Sigma-Aldrich); 1:500 rabbit polyclonal anti-ionized calcium-binding adapter molecule 1 (IBA-1, Wako); 1:300 goat anti-Sox2 (sex determining region Y box-2; R\&D Systems); 1:500 mouse anti-NeuN (Millipore).

The binding of these antibodies was detected over $24 \mathrm{~h}$ at $4^{\circ} \mathrm{C}$ with the following donkey AlexaFluor-conjugated secondary antibodies, as appropriate (1:1000, Invitrogen): donkey anti-rabbit AlexaFluor 594conjugated, donkey anti-rabbit AlexaFluor 555-conjugated (MCM2, Iba1 detection); donkey anti-rat AlexaFluor 488-conjugated (BrdU, CldU detection), donkey anti-goat AlexaFluor 594-conjugated [doublecortin (DCX), Sox2 detection], donkey anti-mouse AlexaFluor 647conjugated (IdU, GFAP, NeuN detection). Sections were counterstained for 10 min with DAPI (1:1000, Calbiochem-EMD).

Samples were mounted in Neo-Mount (Sigma-Aldrich).

\section{Stereological analysis}

An unbiased estimate of all markers, previously described (Mohammad et al., 2018), as a positive cell number in the SGZ of the DG was acquired using every sixth section throughout the rostral-caudal extent of the hippocampus.

Exhaustive counting from the whole right DG using Olympus DSU "fixed cell" Spinning Disk confocal microscope was used for BrdU, CldU, IdU, and MCM2 analysis, under $40 \times$ oil objective.

The optical fractionator was used for Sox $2 /$ GFAP, DCX, and NeuN analysis. The counting was performed from pictures acquired on the Leica SP8 3D, 3-color Stimulated Emission Depletion Laser Scanning Confocal with Time Gating microscope. We acquired nine images in total per animal, which were projections of five to six images taken every $3 \mu \mathrm{M}$, to compose a final stack ( $z$ dimension) of the whole DG. Images were taken sequentially with the confocal microscope. The only exception was the DCX marker, where we performed six images per animal. Positive cells for markers cited above were counted in each $z$ single plane, avoiding double counting as the $3 \mu \mathrm{M}$ distance, allowed us to follow cells along the stacks.

Quantitative analysis was conducted on the images using ImageJ with the "cell counter" plugin. For each slice, the number of positive-labeled cells was manually counted in an area limited to the inner edge of the supra and infrapyramidal blades of the DG, on the whole surface of the subgranular layer (SGL).

For Sox2/GFAP, we counted only the cells with both markers, as a colocalization analysis as previously described (Ortega-Martínez and Trejo, 2015).

Total positive cells in the whole DG were obtained as the sum of the total number of positive cells in each image, multiplied by 6 , corresponding to the fractions used to divide the whole DG.

\section{NeuN analysis}

Imaging of hippocampal cross sections was performed using the Olympus DSU fixed cell Spinning Disk Confocal via a $40 \times$ oil objective. Three images of the granular cell layer (GCL) were randomly taken and analyzed per animal.

Quantitative analysis was conducted on the images using ImageJ with the "cell counter" plugin. For each slice, the number of NeuN-labeled cells was manually counted in an area limited to the supra or infrapyra- midal blades of the DG. Either the area of suprapyramidal or infrapyramidal blades of the DG was also measured using the Image line tool. For each slice, cell density was calculated. We multiplied by $10^{6}$ as previously performed (Ortega-Martínez and Trejo, 2015), for an easy interpretation.

\section{Dendrite analysis and maturation index}

To analyze the total number of DCX-positive cells in each of the animals, pictures of the complete stereological series ( 1 in 9) were taken using a confocal microscope Caliber ID (Imaging \& Diagnostics) RS-G4 Upright Confocal, with the Olympus $40 \times$ UPlanFL N objective, N.A. 1.30 oil for our scans. We acquired six images in total per animal, each of which is a projection of $5 / 6$ images taken every $3 \mu \mathrm{M}$ to compose a final stack $(z$ dimension) of the whole DG. Images were taken sequentially with the confocal microscope. DCX-positive cells were counted in each $z$ single plane, using ImageJ with the "cell counter" plugin, avoiding double counting as the $3 \mu \mathrm{m}$ distance allowed us to follow cells along the stacks. Total DCX-positive cells in the whole DG were obtained as the sum of the total number of DCX in each image, multiplied by 9 , corresponding to the fractions used to divide the whole DG.

In addition, for the analysis of total DCX-positive cells, we performed a separate analysis of DCX-positive cells with dendrites, and DCX-positive cells with tertiary dendrites, to calculate the dendrite maturation index in the newborn neurons cells. The maturation index was then calculated, as previously described in the literature (Quesseveur et al., 2013; Mendez-David et al., 2014; Mohammad et al., 2018; number of DCX-positive cells with tertiary dendrites/number of DCX-positive cells).

\section{Differentiation analysis}

To analyze the differentiation of the newborn cells in the neurogenesis process, we performed colocalization analysis of BrdU (15 d, post injection) with DCX (as a reference marker of neuroblasts) and NeuN (gold standard marker for mature neurons). Results (Fig. $2 E-H$ ) were done as the percentage of the colocalization cells by the BrdU total cells.

\section{Behavior analysis}

Mice were maintained on a $12 \mathrm{~h}$ light/dark cycle and supplied with food and water ad libitum. Behavioral tests were performed between 8:00 A.M. and 15:00 P.M. in the order described below with a $2-3 \mathrm{~d}$ interval between tests. For all procedures, observers were blind to the genotype or treatments.

\section{Marble burying test}

The burying of a marble is a natural defense mechanism in mice that occurs under conditions of stress or states of anxiety. The marble burying assay is able to detect phenotypes related to anxiety and obsessivecompulsive disorders (Angoa-Perez et al., 2013), and it is a test commonly used in AD studies (Torres-Lista et al., 2015). In response to novel bedding/environment, mice exhibit digging behavior. Marbles serve as means to measure the amount of digging.

Mice were individually housed in a cage filled with $5 \mathrm{~cm}$ of novel bedding for a 30 min testing period. Up to 10 mice were examined during each testing round. Before each round, the experimenter evenly spaced 20 marbles across the bedding surface. After $30 \mathrm{~min}$, each mouse was returned to its home cage and all marbles $2 / 3$ buried or more were counted. Borderline marbles $(\sim 2 / 3$ buried or less) were not included in the data analysis.

\section{Light/dark}

This test was performed in the Carlson facilities of the University of Chicago using a box $(30 \times 45 \times 30 \mathrm{~cm})$, partitioned equally, with opaque black walls (dark), white walls without a roof (light), and an opening $(5.5 \times 7 \mathrm{~cm}$ allowing free movement between the compartments) in the partition. Animals were placed in the light part of the arena and monitored using a camcorder (Sony Handycam HDR-CX405 1080P HD) for $10 \mathrm{~min}$. Time spent in the light and dark areas was analyzed manually. The experimenter was blind for the animals analyzed. Other behaviors analyzed were time to escape, number of exits to the light area, rearing, grooming, and number of risk assessments (Mohammad et al., 2018). However, for easier interpretation we have represented only time in light area (s) (see Fig. $5 A, D$ ), number of rearings (Fig. $5 B, E$ ), and number of grooming (Fig. 5C,F) behaviors. 
A

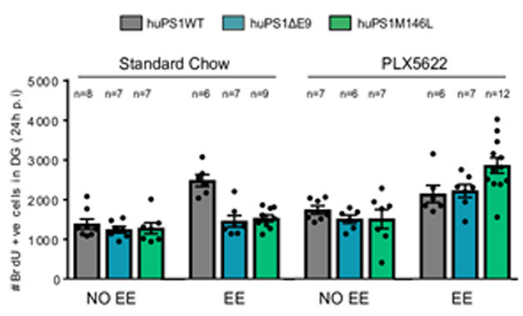

D

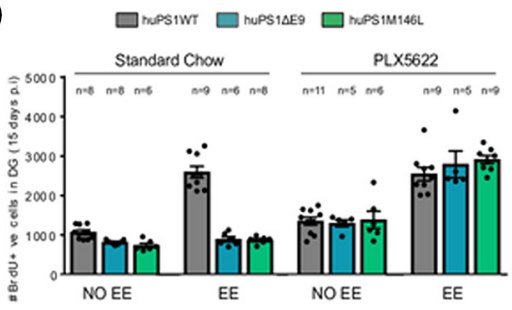

B

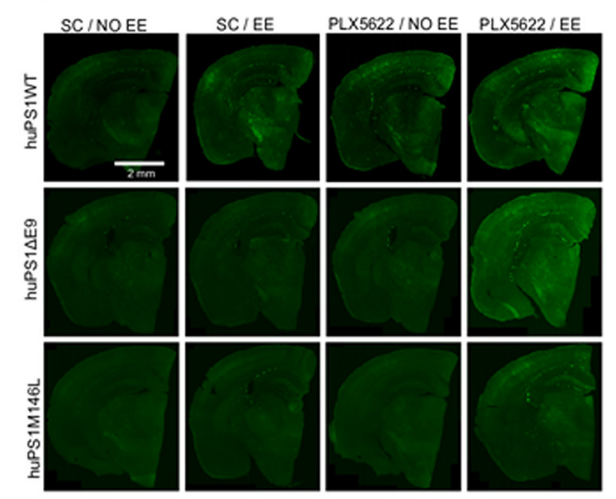

E

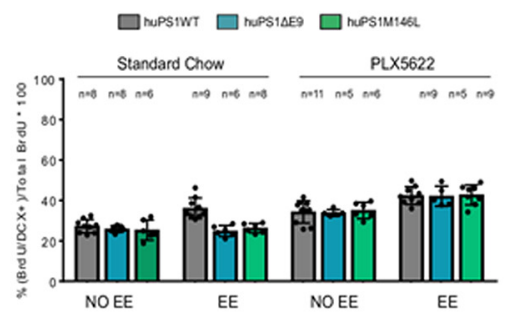

G
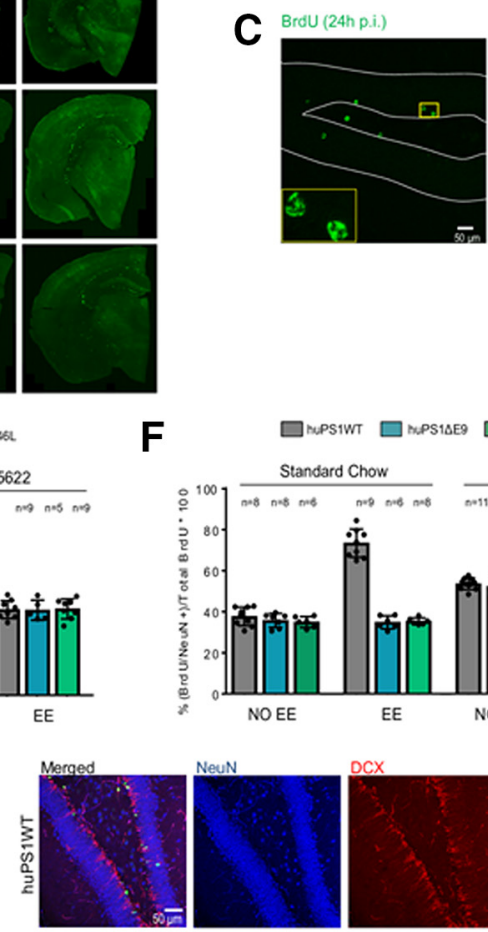

$\mathbf{F}$

$\square$ MUPSWT $\square$ MPS1SEg $\square$ MUPS1M146L
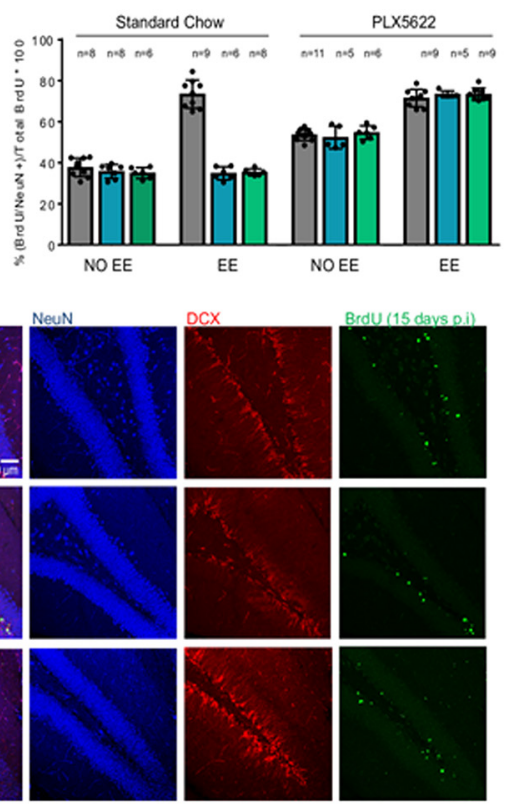

Figure 2. Expression of FAD-linked PS1 variants impairs enrichment-induced proliferation, survival, and differentiation of hippocampal neural progenitors; rescue by microglia depletion. $\boldsymbol{A}$, Proliferation: BrdU-positive cells in DG of mice expressing huPS1WT or FAD-linked huPS1 $\triangle$ E9 and huPS1M146L in SC diet - /+ EE or PLX5622 diet - /+ EE. Three-way ANOVA was significantly different $(p<0.001)$; differences were because of the three individual fixed factors: genotype $(p=0.025)$, diet $(p<0.001)$, and enrichment $(p<0.001)$. In addition, some of the differences found among groups were because of the combination of two factors: genotype $\times$ diet $(p=0.004)$ or all three factors: genotype $\times$ diet $\times$ enrichment $(p=0.001)$. $\boldsymbol{B}$, Representative photomicrographs of the 12 groups of animals in the proliferation study. Images acquired with a CRi Panoramic Scan Whole Slide Scanner at the Microscopy core facilities of the University of Chicago. Tiling was performed to represent whole brains. Pictures were taken using a $20 \times$ objective. Scale bar, $2 \mathrm{~mm}$. C, Detail of BrdU cells within the DG. Pictures were taken using Leica SP8 confocal microscope under 20X objective. Scale bar, $50 \mu \mathrm{m}$. Zoom of BrdU cells is represented. D, Survival: BrdU-positive cells in the DG of mice expressing huPS1WT or FAD-linked huPS1 EE9 and huPS1M146L in SC diet - / EE or PLX5622 diet - / EE 2 weeks after intraperitoneal injection. Three-way ANOVA was significantly different $(p<0.001)$; differences were because of the three individual fixed factors: genotype $(p<0.001)$, diet $(p<0.001)$, and enrichment $(p<0.001)$. Some differences were also because of the combination of different factors, such as genotype $\times$ diet $(p<0.001)$, genotype $\times$ enrichment $(p=0.003)$ and diet $\times$ enrichment $(p<0.001)$. The combination of those three factors, genotype $\times$ diet $\times$ enrichment $(p<0.001)$, was also responsible for differences found in the experiment. $E$, Percentage of BrdU cells that are DCX-positive 2 weeks after injection. Percentage of BrdU-positive, DCX-positive cells in DG of mice expressing huPS1WT or FAD-linked huPS1 $\Delta$ E9 and huPS1M146L inSC diet $-1+$ EE or PLX5622 diet - / + EE. Three-way ANOVA was significantly different $(p<0.001)$. Those differences were because of the three fixed factors genotype $(p=0.005)$, diet $(p<0.001)$, and enrichment $(p<0.001)$, but also because of the combination of factors such as follows: genotype $\times$ diet $(p=0.005)$ and diet $\times$ enrichment $(p=0.008)$. There was a trend toward significance in the combination of genotype $\times$ enrichment $(p=0.054)$ and in the combination of the three factors, genotype $\times$ diet $\times$ enrichment $(p=$ 0.059). $\boldsymbol{F}$, Percentage of BrdU cells that are NeuN-positive 2 weeks after injection. Percentage of BrdU-positive, NeuN-positive cells in DG of mice expressing huPS1WT or FAD-linked huPS1 $\Delta$ E9 and huPS1M146L in SC diet - / EE or PLX5622 diet - / + EE. Three-way ANOVA was significantly different $(p<0.001)$, and those differences are because of our three independent fixed factors: genotype $(p<0.001)$, diet $(p<0.001)$, and enrichment $(p<0.001)$; but also, because of the combination of the different factors: genotype $\times$ diet $(p<0.001)$, genotype $\times$ enrichment $(p<$ $0.001)$ and diet $\times$ enrichment $(p<0.001)$. The combination of three factors also contributed to the differences between groups genotype $\times$ diet $\times$ enrichment $(p<0.001)$. $\mathbf{G}$, Representative photomicrographs of the three transgenic lines in PLX5622 diet and EE conditions used in the differentiation experiment. Pictures were taken using Leica SP8 confocal microscope under $20 \times$ objective. Scale bar, $50 \mu \mathrm{m}$. $\boldsymbol{H}$, High-resolution image of BrdU/DCX/NeuN-labeled cells in the DG. Image acquired with a Leica SP8 confocal microscope under $40 \times$ objective. Scale bar, $15 \mu \mathrm{m}$.

\section{Statistical analysis}

The data were analyzed using a linear model, with "genotype", "diet", and "enrichment" as fixed factors, and the corresponding parameter analyzed (BrdU, Sox2/GFAP, MCM2, DCX, NeuN, etc.) as the dependent variable, in a three-way ANOVA analysis. SPSS 17.0.1 software (Apache Software Foundation) was used to perform this statistical analysis. Microsoft Excel v2016 was used for the blind analysis, calculations and to create the raw data posteriorly used in SPSS. GraphPad Prism software was used for graphs generations. Tukey and Bonferroni post hoc tests were performed. For correlation analysis, Pearson correlation analysis was performed using $\mathrm{R}$ software. The tests for significance of the correlations were corrected for multiple comparisons within each study using the Bonferroni procedure, which controls familywise error rate at 0.05 . In all cases, results were considered statistically significant when $p<$ 
0.05 , yet a $p$ value in the interval $0.1>p \geq 0.05$ was considered as a trend toward significance. All statistical analyses performed and $p$ values obtained for each experiment are explained in the results sections and within their corresponding figure legend.

\section{Results \\ PLX5622, a selective CSF1R-kinase inhibitor depletes microglia in transgenic mice expressing human WT PS1 or FAD-linked PS1 variants}

To assess the role of microglia in the transgenic mouse models used in this study (Fig. 1A,B), we used an established strategy to deplete microglia in brain. It is well established that microglia are generated from the yolk sac, and colonize the CNS during early development through the action of the colony-stimulating factor 1 (CSF1) that regulates proliferation, differentiation, and function of macrophage lineage cells through binding to its specific receptor, CSF1R (Patel and Player, 2009). Under physiological conditions, microglia are the only cells in the CNS that express CSF1R (Erblich et al., 2011) and PLX5622, a potent inhibitor of CSF1R tyrosine kinase activity $(\mathrm{KI}=5.9 \mathrm{nM})$ with at least 50 -fold selectivity over 4 related kinases, and over 100 -fold selectivity against a panel of 230 kinases, depletes $>98 \%$ of microglia in mice treated for $7 \mathrm{~d}$ (1200 ppm in chow corresponding to $\sim 185$ $\mathrm{mg} / \mathrm{kg}$ body weight; Dagher et al., 2015). Moreover, microglia elimination lead to no discernable deficits in behavior or learning and memory in the tasks tested (Dagher et al., 2015). Here, we show that treating our three mouse lines that express prion protein $(\mathrm{PrP})$ promoter-driven huPSEN1WT or the FAD-linked huPSEN1 $\triangle E 9$ and $h u P S E N 1 M 146 L$ transgenes for $7 \mathrm{~d}$ resulted in the near complete depletion ( $99 \%$ ) of microglia in the DG of the hippocampus compared with mice fed with standard chow diet (SC; Fig. 1B). To avoid microglia repopulation, PLX5622 groups were fed with this specific diet during the entire experiment, as explained in Figure $1 A$.

\section{PLX5622 depletes microglia in PS1 $1^{\text {M146V/+ }}$ mice}

One caveat of the studies reported above is that transgenes encoding huPS1WT or the FAD-linked PS1 variants are transcriptionally driven by the ubiquitously-expressed mouse PrP promoter. Hence, it can be argued that transgene-driven PS1 expression levels in specific cell types do not accurately represent the cell-specific expression of endogenous PS1. However, PS1 has been shown to be expressed endogenously in neurons (Elder et al., 1996; Lah et al., 1997), cerebral vasculature (Lah et al., 1997), glia, and oligodendrocytes (Lah et al., 1997; Lee et al., 1997). Furthermore, we have demonstrated that PrP promoter-driven transgene-mediated "overexpression" of either wild-type or FAD-linked human PS1 does not elevate the total levels of PS1, a phenomenon that is the consequence of the association of PS1 with limiting cellular factors that ultimately leads to the "replacement" of endogenous PS1 and accumulation of human PS1 to endogenous levels (Thinakaran et al., 1997). In any event, and to allay concerns pertaining to overexpression and/or cell-typespecific expression patterns in the transgenic lines that might not accurately reflect the expression patterns of endogenous PS1, we then assessed the impact of mutant PS1 on EE-induced AHNPC phenotypes when expressed under physiological levels. For this, we exposed adult mice that constitutively express the PSEN1 M146V mutation driven by the endogenous PSEN1 promoter $\left(P S 1^{M 146 /++}\right.$ ) mice (Guo et al., 1999) to standard or EE-housed conditions. The experimental design for this group is described in Figure $1 C, D$. As described previously, all mice receiving the PLX5622 diet for $7 \mathrm{~d}$ showed a $\sim 99 \%$ depletion of microglia in the DG of the hippocampus. Again, mice were kept on the PLX5622 diet for the entirety of the experiment (Fig. 1D).

\section{Microglial depletion restores deficits in proliferation and survival of AHNPCs in mice expressing FAD-linked variants following EE}

We previously demonstrated that the proliferation and neuronal differentiation of AHNPCs is compromised in the hippocampus of mice expressing FAD-linked variants compared with mice expressing huWTPS1 (Choi et al., 2008). To assess the influence of microglia on AHNPC proliferation, we fed cohorts of 4 -week-old male mice with either PLX5622 or control diet for 1 week. After the first week, the PLX5622 cohort was exposed to SH or EE conditions for an additional 4 weeks in the presence of PLX5622. In parallel, the control diet cohorts were exposed to $\mathrm{SH}$ or $\mathrm{EE}$ conditions for an additional 4 weeks in the presence of control diet.

Animals were then injected with a single bolus of BrdU intraperitoneally, then killed after $24 \mathrm{~h}$. In parallel, "control” cohorts of mice were exposed mice to SH or EE conditions for 4 weeks, injected with a bolus of BrdU and then killed after $24 \mathrm{~h}$. Brain sections were subject to immunofluorescence analysis using a BrdU-specific antibody; the number of BrdU-positive cells in the SGL and GCL was quantified by stereological methods. As we previously reported (Choi et al., 2008), we observed no differences in proliferation of BrdU-labeled AHNPCs in the three mouse lines that were maintained in SH conditions with SC. However, when we exposed animals to EE, huPS1WT mice showed an increase in proliferation, but this was not the case in mice expressing the FAD-linked huPS1 $\Delta \mathrm{E} 9$ or huPS1M146L variants (Fig. $2 A-C$ ), findings consistent with our previous observations (Veeraraghavalu et al., 2013). Remarkably, animals that were fed with PLX5622 demonstrated a complete restoration of the proliferation deficit observed in EE mice expressing mutant PS1; the EE-mediated proliferation of AHNPCs was no different between the three mouse lines (Fig. 2A-C).

For proliferation study, Three-way ANOVA was significantly different $(p<0.001)$; differences were because of the three individual fixed factors: genotype $(p=0.025)$, diet $(p<0.001)$, and enrichment $(p<0.001)$. In addition, some of the differences found among groups were because of the combination of two factors: genotype $\times \operatorname{diet}(p=0.004)$ or all three factors: genotype $\times$ diet $\times$ enrichment $(p=0.001)$.

To examine the impact of microglia on survival of AHNPCs, animals were fed with PLX5622 for 1 week and then exposed to $\mathrm{SH}$ or EE conditions for 2 weeks in the presence of PLX5622. Animals were then injected with a bolus of BrdU and returned to either SH or EE conditions with PLX5622 in the diet for two additional weeks, then killed. In parallel, cohorts of control animals were exposed to $\mathrm{SH}$ or EE conditions for 2 weeks, then injected with a bolus of BrdU, then returned to SH or EE conditions for 2 weeks, then killed. We observed an elevation in BrdUlabeled AHNPCs that survive in huWTPS1 mice following EE, but again, this was not observed in enriched mice expressing the PS1 variants. However, PLX5622 treatment fully restored BrdUpositive cells in EE mice expressing PS1 variants to the levels observed in enriched huWTPS1 mice (Fig. 2D). For survival study, Three-way ANOVA was significantly different $(p<$ $0.001)$; differences were because of the three individual fixed factors: genotype $(p<0.001)$, diet $(p<0.001)$, and enrichment $(p<0.001)$. Some differences were also because of the combination of different factors, such as genotype $\times \operatorname{diet}(p<0.001)$, genotype $\times$ enrichment $(p=0.003)$, and diet $\times$ enrichment 
$(p<0.001)$. The combination of those three factors, genotype $\times$ diet $\times$ enrichment $(p<0.001)$, was also responsible for differences found in the experiment.

To assess the differentiation of BrdU-labeled AHNPCs, we used the animals that were examined for the survival studies (Fig. $2 D)$. In this case, we performed double-labeling with antibodies against BrdU and either the neuroblast marker DCX or the neuronal marker NeuN, which labels the nuclear splicing factor Fox-3 (Kim et al., 2009). Although the levels of BrdU/DCXpositive cells in the SGL of the DG were similar across all genotypes in SH and SC conditions, EE significantly elevated the numbers of BrdU/DCX-positive cells in huWTPS1 mice, but not in mice expressing the FAD-linked variants (Fig. 2E, $G, H$ ). The PLX5622 diet slightly increased the total numbers of BrdU/DCXpositive cells in all genotypes and to similar levels, and the PLX5622 diet with EE elevated the numbers of BrdU/DCXpositive cells to even higher levels, but these levels were similar across all genotypes (Fig. 2E, $G, H$ ). For BrdU/DCX analysis, three-way ANOVA was significantly different $(p<0.001)$. Those differences were because of the three fixed factors: genotype ( $p=$ $0.005)$, diet $(p<0.001)$, and enrichment $(p<0.001)$; but also because of the combination of factors such as follows: genotype $X$ $\operatorname{diet}(p=0.005)$ and diet $\times$ enrichment $(p=0.008)$. There was a trend toward significance in the combination of genotype $\times$ enrichment $(p=0.054)$ and in the combination of the three factors, genotype $\times$ diet $\times$ enrichment $(p=0.059)$.

We then examined the fraction of BrdU/NeuN-positive cells in the DG in SH/SC and observed that the levels of newly-born progenitors that had matured to granule cells were similar across all genotypes. On the other hand, and as we had reported earlier (Choi et al., 2008), EE only elevated the fraction of BrdU/NeuNpositive cells in huWTPS1 mice, but not in mice expressing the FAD-linked variants (Fig. $2 F-H$ ). The PLX5622 diet increased the fraction of BrdU/NeuN-positive cells in all genotypes and to similar levels, and the PLX5622 diet with EE elevated the fraction of BrdU/DCX-positive cells even further. Importantly, the fraction of BrdU/NeuN-positive cells were indistinguishable across all genotypes. Hence, we can conclude that PLX5622mediated microglial depletion restores the deficits in EEmediated AHNPC proliferation, production, and phenotypes of DCX-positive neuroblasts and neuronal maturation that are observed in mice expressing FAD-linked PS1 variants. For BrdU/NeuN analysis three-way ANOVA was significantly different $(p<0.001)$, and those differences are because of our three independent fixed factors: genotype $(p<0.001)$, diet $(p<0.001)$, and enrichment $(p<0.001)$; but also, because of the combination of the different factors: genotype $\times \operatorname{diet}(p<$ $0.001)$, genotype $\times$ enrichment $(p<0.001)$, and diet $\times$ enrichment $(p<0.001)$. The combination of three factors also contributed to the differences between groups genotype $\times$ diet $\times$ enrichment $(p<0.001)$.

Collectively, these studies reveal that microglial depletion fully rescues the deficits in proliferation and survival of AHNPCs, and the differentiation of newly-born AHNPCs into DCXpositive neuroblasts and NeuN-positive mature neurons in the dentate gyrus of mice expressing FAD-linked PS1 variants following EE.

\section{Microglial depletion restores steady-state levels of AHNPCs, transit amplifying progenitors and mature neurons in mice expressing FAD-linked PS1 following EE}

Having demonstrated that PLX5622-mediated depletion of microglia rescues the deficits in levels of proliferation, survival, and differentiation of BrdU-labeled AHNPCs in mice expressing FAD-linked PS1 variants, we further determined the steady-state levels of AHNPCs located in the SGL of the DG by assessing the colocalization of antibodies raised against SRY (Sox2 and GFAP), as previously described (Ortega-Martínez and Trejo, 2015). The levels of Sox2/GFAP-positive neural stem cells (NSCs) in the DG of all three transgenic lines housed in $\mathrm{SH}$ and SC were comparable (Fig. $3 A, B$ ). However, when exposed to EE, we observed an increase in NSCs in huWTPS1 mice, but EE failed to increase the levels of these cells in mice expressing the FAD-linked variants. Interestingly, PLX5622-mediated depletion of microglia in mice housed in SH conditions led to a slight elevation of NSCs in all transgenic lines, but to a similar extent. Importantly, when fed PLX5622 chow and housed in EE conditions, the levels of NSCs increased to even higher levels in all transgenic lines, with no apparent difference in total numbers between lines (Fig. 3A,B). Three-way ANOVA analysis was significantly different between groups. Those differences are because of the three individual factors: genotype $(p=0.000)$, diet $(p<0.001)$, and environmental enrichment $(p=0.001)$.

We then examined the steady-state levels of amplifying progenitors using antibodies to the transit amplifying cell marker, DNA replication licensing factor MCM2 (Ortega-Martínez and Trejo, 2015). We did not observe any differences in MCM2positive cells between the mouse lines in mice housed in $\mathrm{SH}$ and SC, but upon exposure to EE, the levels of amplifying progenitors were elevated only in huWTPS1 mice, but not in mice expressing the FAD-linked variants (Fig. $3 C, D$ ). On the other hand, whereas PLX5622 led to an elevation in MCM2-positive cells in all mouse lines in SH conditions, the numbers of these cells were elevated to even higher levels with PLX5622 and EE. Importantly, we did not observe a difference in MCM2-positive cells between the mouse lines in the presence of PLX5622 and subject to EE. Three-way ANOVA of MCM2 analysis showed a significant difference $(p<$ $0.001)$. These differences are because of the three individual factors: genotype $(p=0.000)$, diet $(p<0.001)$, and environmental enrichment $(p<0.001)$. In addition, the combination of these factors was significantly different when considering genotype $x$ $\operatorname{diet}(p<0.001)$, genotype $\times$ enrichment $(p=0.012)$, and diet $\times$ enrichment $(p<0.001)$ and there was a trend toward significance for the combination of genotype $\times \operatorname{diet}(p=0.066)$. Finally, the combination of our three independent factors genotype $\times$ diet $\times$ environmental enrichment was also significantly different $(p<0.001)$.

Finally, we then assessed the total level of mature neurons in the DG using anti-NeuN (Fig. $3 E, F$ ). Similar to the results obtained with anti-MCM2-specific antibodies, we did not observe any differences in total NeuN-positive cells in the DG of all three mouse lines in SC and SH or SC and EE. However, the DG of all lines of mice fed with PLX5622 exhibited elevated levels of NeuNpositive cells but the levels were no different between lines. Three-way ANOVA analysis was significant $(p<0.001)$ and all differences between groups are just because of diet $(p<0.001)$, whereas the other individual fixed factors and all the possible combination between factors were not significantly different ( $p>0.05$ in all cases).

Extending these analyses, we finally evaluated the neuroblasts and dendritic morphology of DCX-positive cells, as previously described (Plümpe et al., 2006). Three different parameters, presented in Figure 3, include: total DCX-positive cells (Fig. 3G), DCX-positive cells with dendrites (Fig. $3 H$ ), and the "maturation index" of those cells (Fig. 3I-K), as previously reported (Quesseveur et al., 2013; Mendez-David et al., 2014; Mohammad 
A
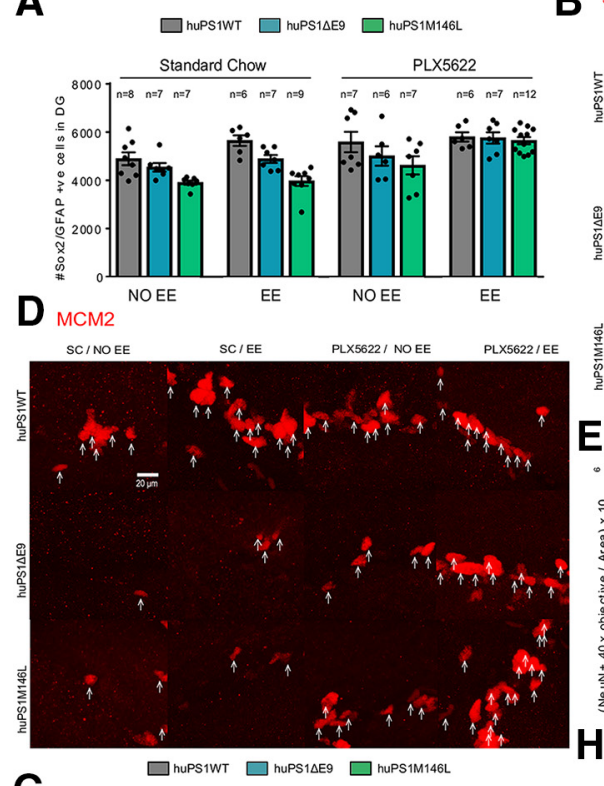

G
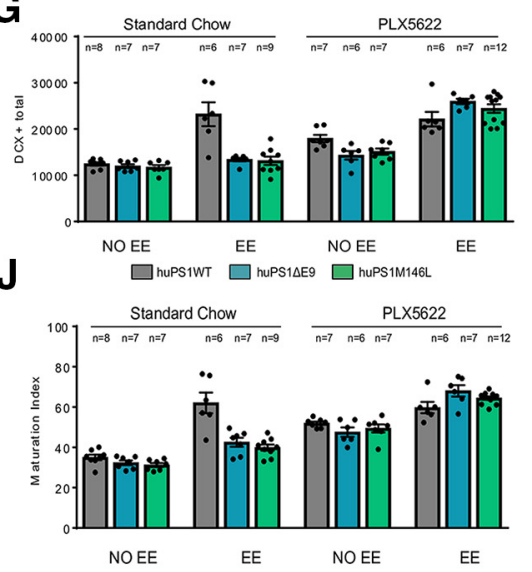

B
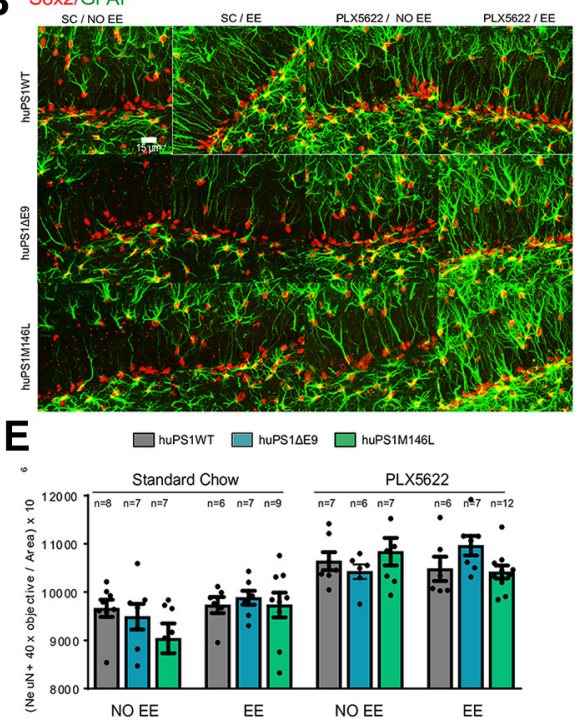

$\square$ huPS1WT $\square$ huPS1 $\square$ hUPS1M146L

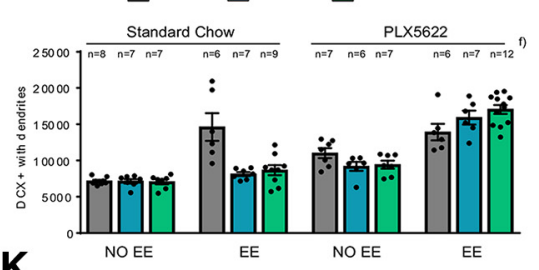

K $\mathrm{DCX} / \mathrm{BrdU}$

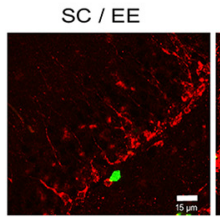

C

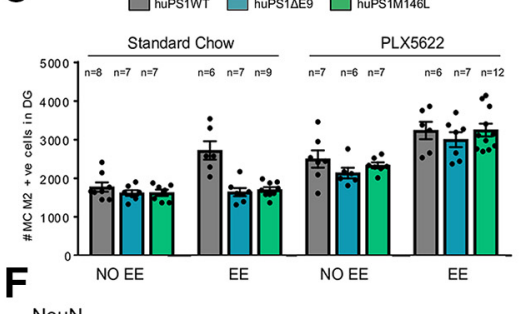

Neun

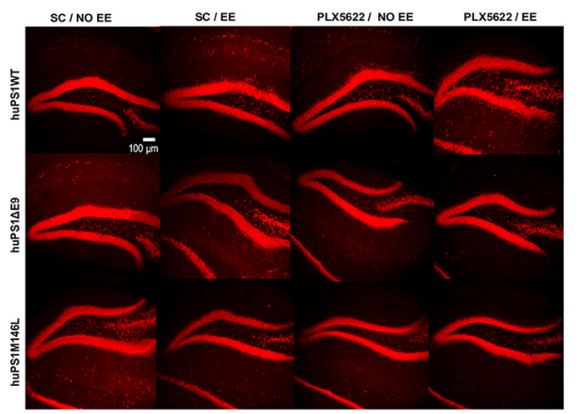

Figure 3. Analysis of neurogenic cell populations. $A$, Sox2/GFAP-positive cells in DG. Numbers of neural stem cells in DG of mice expressing huPS1WT or FAD-linked huPS1 $\triangle$ E9 and huPS1M146L in SC diet - / EE or PLX5622 diet - I+ EE. Three-way ANOVA analysis was significantly different between groups. Those differences are because of the three individual factors: genotype $(p=0.000)$, diet $(p<0.001)$, and environmental enrichment $(p=0.001) \cdot \boldsymbol{B}$, Representative images of Sox2/GFAP-positive neural stem cells in the 12 experimental groups shown in $\boldsymbol{A}$. Micrographs were taken using a Leica SP8 confocal microscope under 40× objective. Scale bar, $15 \mu \mathrm{m}$. C, MCM2-positive cells in DG. Numbers of transit amplifying progenitors in DG of mice expressing huPS1WT or FAD-linked huPS1 $\Delta$ E9 and huPS1M146L in SC diet $-/+$ EE or PLX5622 diet $-/+$ EE. Three-way ANOVA of MCM2 analysis showed a significant difference $(p<0.001)$. These differences are because of the three individual factors: genotype $(p=0.000)$, diet $(p<0.001)$, and environmental enrichment $(p<0.001)$. In addition, the combination of these factors was significantly different when considering genotype $\times \operatorname{diet}(p<0.001)$, genotype $\times$ enrichment $(p=0.012)$, and diet $\times$ enrichment $(p<0.001)$ and there was a trend toward significance for the combination of genotype $\times$ diet $(p=0.066)$. Finally, the combination of our three independent factors genotype $\times$ diet $X$ environmental enrichment was also significantly different $(p<0.001)$. $\boldsymbol{D}$, Representative pictures of MCM2-positive transit amplifying progenitors in the 12 groups shown in $\boldsymbol{C}$. Micrographs acquired with a Leica SP8 confocal microscope under $63 \times$ objective. Scale bar, $20 \mu \mathrm{m}$. E, NeuN-positive cells in DG. Mature neurons in DG of mice expressing huPS1WT or FAD-linked huPS1 $\triangle$ E9 and huPS1M146L in SC diet $-1+$ EE or PLX5622 diet $-1+$ EE. Three-way ANOVA analysis was significant $(p<0.001)$ and all differences between groups are just because of diet $(p<0.001)$, whereas the other individual fixed factors and all the possible combination between factors were not significantly different ( $p>0.05$ in all cases). $\boldsymbol{F}$, Representative images of NeuN-positive mature neurons in the 12 groups shown in $\boldsymbol{E}$. Images acquired using a Leica Sp8 confocal microscope under $10 \times$ objective. Scale bar, $100 \mu \mathrm{m}$. G, Total numbers of DCX-positive cells. Neuroblasts in DG of mice expressing huPS1WT or FAD-linked huPS1 $\Delta$ E9 and huPS1M146L in SC diet $-/+$ EE or PLX5622 diet $-/+$ EE. Three-way ANOVA showed a significant difference $(p<0.001)$. These differences are because of genotype $(p<0.001)$, diet $(p<0.001)$, and enrichment $(p<0.001)$. In addition, the combination of diet $\times$ enrichment was also significant ( $p=0.002$ ). Finally, the combination of our three fixed factors was also responsible for the differences found in our groups: genotype $\times$ diet $X$ enrichment $(p<0.001)$. $\boldsymbol{H}, \mathrm{DCX}$-positive cells with dendrites. Three-way ANOVA showed the groups were significantly different $(p=0.000)$ and these differences were because of our three independent factors: genotype $(p=0.018)$, diet $(p<0.001)$, and enrichment $(p<0.001)$. In addition, some of the differences observed between groups were because of the combination of those fixed factors, such as genotype $\times \operatorname{diet}(p=0.001)$, diet $\times$ enrichment $(p=0.008)$ and the combination of the three independent factors genotype $\times$ diet $\times$ enrichment $(p<0.001)$. $\boldsymbol{I}$, Representative images of DCX-positive cells in the DG of the 12 experimental groups shown in $\boldsymbol{G}$ and $\boldsymbol{H}$. Pictures were acquired with a Leica SP8 confocal microscope, under $10 \times$ objective. Scale bar, $50 \mu \mathrm{m}$. J, Maturation index of DCX-positive cells. Calculation of the ratio of DCX cells with tertiary dendrites to total $D C X$ cells. Three-way ANOVA was significantly different $(p<0.001)$. These differences, in this case, were because of the individual three fixed factors, genotype $(p<0.001)$, diet $(p<0.001)$, and enrichment $(p<0.001)$, and the combination of the following factors: genotype $\times$ diet $(p<0.001)$. Finally, the combination of the three factors also contributed to the final output observed in our experiment: genotype $\times$ diet $\times$ enrichment $(p<0.001) . K$, Micrographs of $D C X+$ cells were to highlight the complexity of their dendrites. Pictures acquired with a Leica SP8 confocal microscope, under $40 \times$ objective. Scale bar, $15 \mu \mathrm{m}$. 
et al., 2018). It is widely assumed the maturation index, or in other words, the complexity of dendritic arborization, is directly related to the functioning of those newborn neurons into the hippocampal network (Llorens-Martín et al., 2015). Thus, this parameter provides an indirect approximation of the functionality of those newborn cells (Llorens-Martín et al., 2015). In SC and $\mathrm{SH}$, the three mouse lines exhibited similar cell levels of DCXpositive cells in the different stages of development, and similar maturation indices. However, EE lead to an increase in all these parameters in only the huPS1WT mouse line fed with SC diet, whereas both transgenic lines expressing the FAD-PS1 variants did not exhibit an elevation in cell numbers or tertiary dendrite arborization. On the other hand, transgenic mice expressing the FAD-linked PS1 variants fed with PLX5622 and subject to EE restored the levels of DCX-positive cells, dendritic arborization, and maturation index to levels no different to those seen in mice expressing huWTPS1. For DCX total analysis, three-way ANOVA showed a significant difference $(p<0.001)$. These differences are because of genotype $(p<0.001)$, diet $(p<0.001)$, and enrichment $(p<0.001)$. In addition, the combination of diet $\times$ enrichment was also significant $(p=0.002)$. Finally, the combination of our three fixed factors was also responsible for the differences found in our groups: genotype $\times$ diet $\times$ enrichment $(p<0.001)$. In the case of DCX with dendrites, three-way ANOVA showed the groups were significantly different $(p=$ 0.000 ) and these differences were because of our three independent factors: genotype $(p=0.018)$, diet $(p<0.001)$, and enrichment $(p<0.001)$. In addition, some of the differences observed between groups were because of the combination of those fixed factors, such as genotype $\times \operatorname{diet}(p=0.001)$, diet $\times$ enrichment $(p=0.008)$, and the combination of the three independent factors genotype $\times$ diet $\times$ enrichment $(p<0.001)$. Finally, in the analysis of the maturation index, three-way ANOVA was significantly different $(p<0.001)$. These differences, in this case, were because of the individual three fixed factors, genotype $(p<$ $0.001)$, diet $(p<0.001)$, and enrichment $(p<0.001)$, and the combination of the following factors: genotype $\times \operatorname{diet}(p<$ 0.001). Finally, the combination of the three factors also contributed to the final output observed in our experiment: genotype $X$ diet $\times$ enrichment $(p<0.001)$.

Thus, we conclude that in mice expressing FAD-liked PS1, microglial depletion restores the steady-state levels of Sox/GFPpositive AHNPCs, MCM2-positive transit-amplifying progenitors, neuroblasts, and NeuN-positive mature neurons to the levels observed in huWTPS1 mice following EE.

\section{The deficits in EE-mediated proliferation and survival of AHNPCs in PS1 ${ }^{\mathrm{M146V/+}}$ mice is mediated by microglia}

To assess the impact of microglia on the proliferation and survival of AHNPCs in mice that express FAD-linked PS1 at physiological levels and cellular distributions, we exposed adult mice that constitutively express the PSEN1 M146V mutation driven by the endogenous $P S 1^{M 146 V /+}$ mice (Guo et al., 1999) to standard or EE-housed conditions. We previously reported that compared with wild-type (nontransgenic) littermates, AHNPCs in mice carrying heterozygous $\left(P S 1^{M 146 V /+}\right)$ or homozygous $\left(P S 1^{M 146 V / M 146 V}\right)$ mutant alleles failed to exhibit EE-induced proliferation and commitment toward neurogenic lineages (Veeraraghavalu and Sisodia, 2013).

Furthermore, the survival of newborn progenitors is diminished in both mice with either heterozygous and homozygous PSEN1 M146V alleles exposed to EE conditions compared with respective EE nontransgenic littermate controls (Veeraraghavalu and Sisodia, 2013). Hence, and in view of our studies (Figs. 2, 3), showing that microglia play a critical role in EE-induced proliferation, differentiation, and survival of AHNPCs in mice expressing transgene-encoded FAD-linked PS1, we felt it was essential to address the possibility that microglia may also play an essential role in these processes in mice with PSEN1 M146V alleles. Interestingly, Lee et al. (2002) had previously demonstrated that microglia isolated from $P S 1^{M 146 V /+}$ mice exhibit marked hypersensitivity to activation by lipopolysaccharide compared with microglia obtained from wild-type mice.

We chose to focus on heterozygous $P S 1^{M 146 V /+}$ mice as this genetic setting would be comparable to the situation in humans where only one copy of the mutant gene is necessary to cause AD. We also altered the experimental strategy to allow for a multiple birth-dating analysis of newborn cells that would allow for analysis of both proliferation and survival in the same animals (Ortega-Martínez and Trejo, 2015); this approach was used previously using the thymidine analogs CldU and IdU (OrtegaMartínez and Trejo, 2015). Similar to results obtained from the transgenic mice expressing FAD-linked variants, the $P S 1^{M 146 V /+}$ mice exhibited similar levels of AHNPC proliferation (Fig. 4A, $B$ ) and survival (Fig. $4 C, D$ ) to nontransgenic littermates in $\mathrm{SH}$ and SC conditions. However, in $P S 1^{M 146 V /+}$ mice, EE did not led to an increase in AHNPC proliferation (Fig. $4 A, B$ ) and survival as was observed in nontransgenic mice (Figs. $4 C, D$ ). Importantly, PLX5622-mediated microglial depletion restored the deficits in proliferation and survival of AHNPCs in $P S 1^{M 146 V /+}$ mice to levels observed in nontransgenic mice (Fig. $4 A, B$ and $C, D$, respectively). In this proliferation study, three-way ANOVA analysis was significantly different $(p<0.001)$. These differences were because of the three individual fixed factors: genotype $(p<$ $0.001)$, diet $(p<0.001)$, and enrichment $(p<0.001)$. In addition, some of the differences found among groups were because of the combination of two factors: genotype $\times$ diet $(p<$ $0.001)$, genotype $\times$ enrichment $(p<0.001)$ or all three factors: genotype $\times$ diet $\times$ enrichment $(p<0.001)$. In the survival study, three-way ANOVA analysis was significantly different $(p<0.001)$. These differences were because of the three individual fixed factors: genotype $(p<0.001)$, diet $(p<$ $0.001)$, and enrichment $(p<0.001)$. Some differences were also because of the combination of different factors, such as genotype $\times \operatorname{diet}(p<0.001)$, and genotype $\times$ enrichment $(p<0.001)$. The combination of these three factors, genotype $\times$ diet $\times$ enrichment $(p=0.010)$, was also responsible for differences found in the experiment.

Moreover, we were also interested in examining the impact of microglia depletion on the total density of neural stem cells (Fig. $4 E, F$ ), neuroblasts (Fig. 4G,H) and mature neurons (Fig. 4I,J). In the case of Sox2/GFAP analysis, three-way ANOVA analysis was significantly different between groups. These differences are because of the three individual factors: genotype $(p=0.000)$, diet $(p<0.001)$, and environmental enrichment $(p=0.001)$. Some differences were also because of the combination of different factors, such as genotype $\times \operatorname{diet}(p<0.0012)$ and genotype $\times$ enrichment $(p=0.0052)$. The combination of these three factors, genotype $\times$ diet $\times$ enrichment $(p<0.0011)$, was also responsible for differences found in the experiment. For DCX analysis, three-way ANOVA showed a significant difference $(p<$ $0.001)$. These differences are because of genotype $(p<0.001)$, $\operatorname{diet}(p<0.001)$, and enrichment $(p<0.001)$. In addition, the combination of genotype $\times \operatorname{diet}(p=0.0029)$ and genotype $\times$ enrichment ( $p=0.0196$ ) was also significant. Finally, the combination of the three fixed factors was also responsible for the differences found in our groups: genotype $\times$ diet $\times$ enrichment 
A

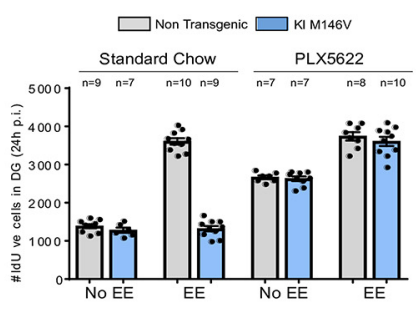

B

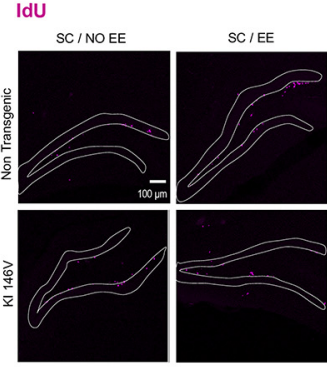

$G^{D C X}$
C

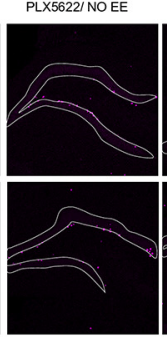

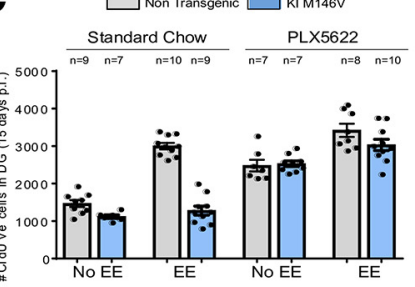

D
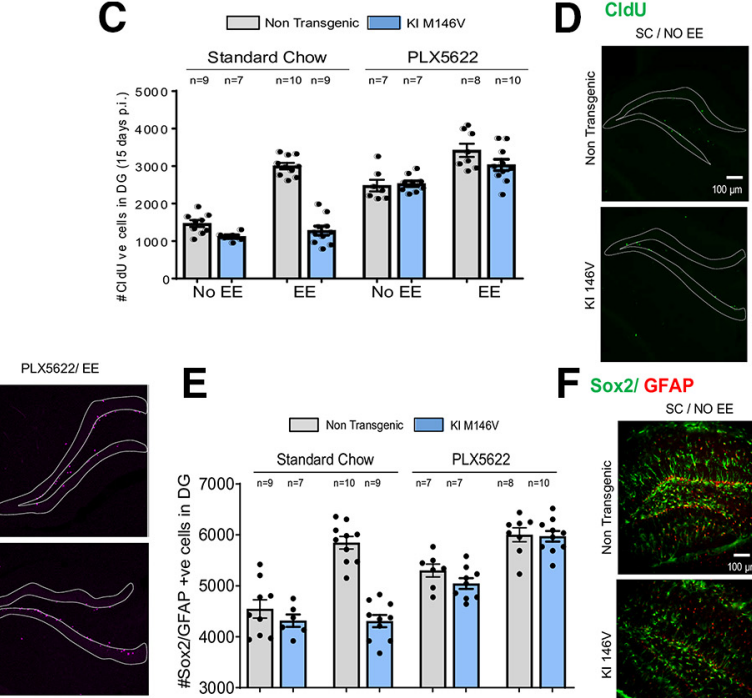

F Sox2/ GFAP

E

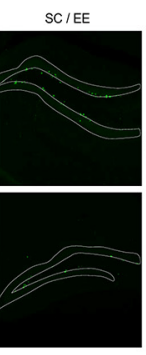

$\mathrm{SC} / \mathrm{EE}$
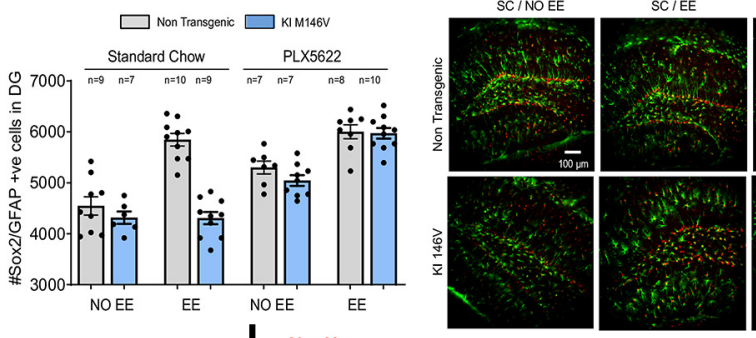

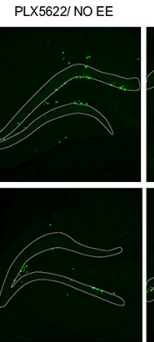

PLX56221

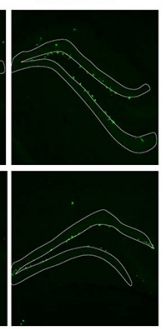

PIX5622/NOEE
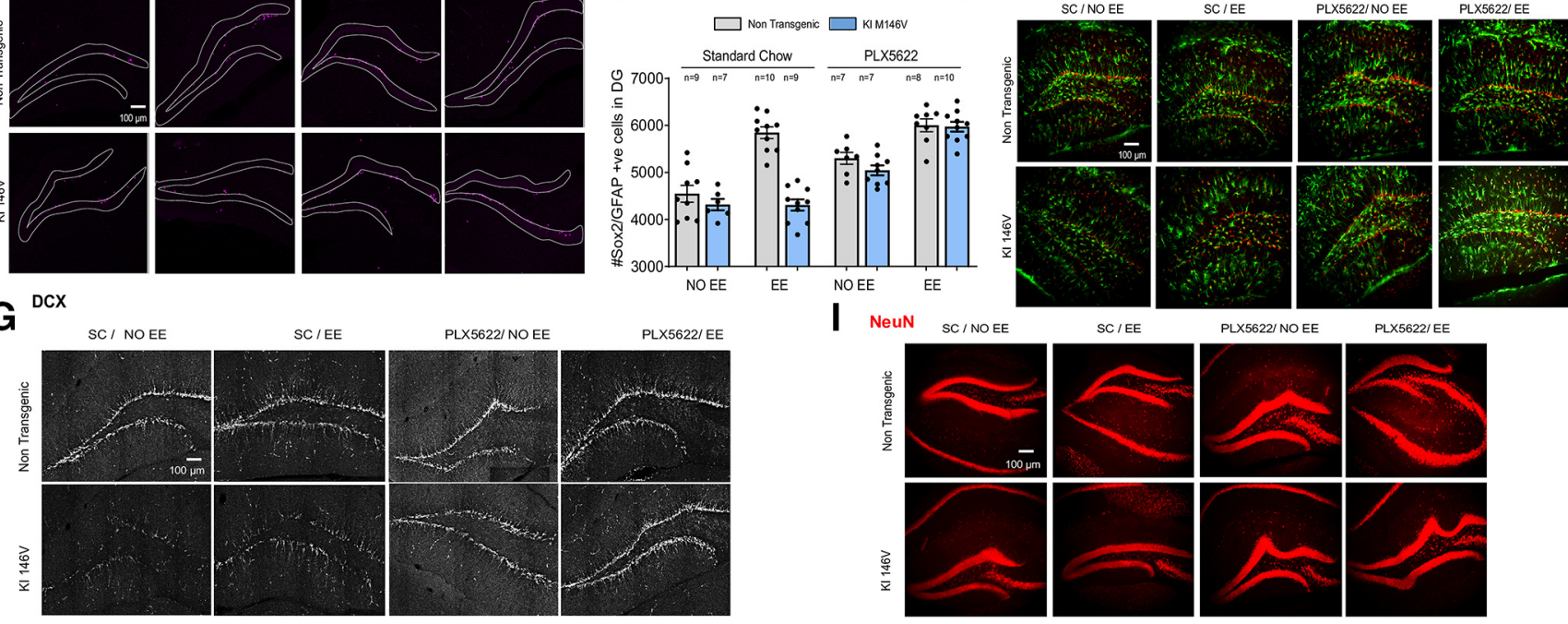

H

$\square$ Non Transgenic $\square$ KI M146V

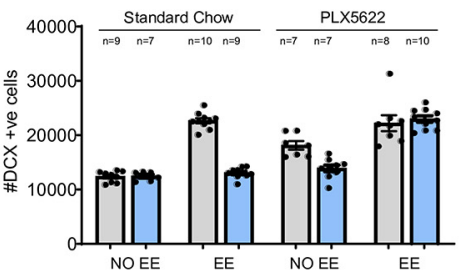

I Neun SCINOEE

PLX5622/ NOEE

PLX5622/EE
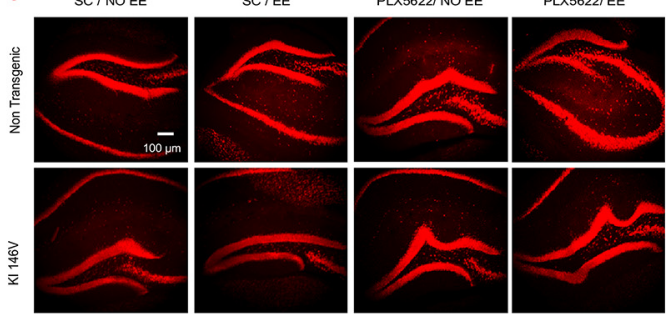

J

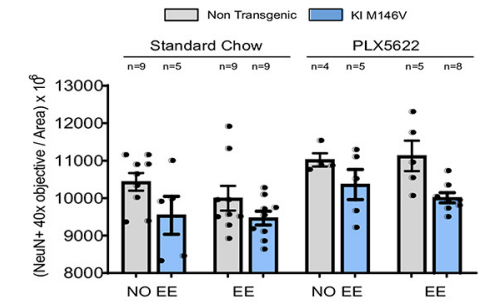

Figure 4. Proliferation, survival and neurogenic cell population studies in nontransgenic and PS1 ${ }^{\mathrm{M} 146 \mathrm{~V} /+}$ mice. $A$, Proliferation IdU-labeled cells in DG of nontransgenic and PS1 ${ }^{\mathrm{M} 146 \mathrm{~V} /+}$ mice in SC diet - / EE or PLX5622 diet - / EE $24 \mathrm{~h}$ after intraperitoneal injection. Three-way ANOVA analysis was significantly different $(p<0.001)$. These differences were because of the three individual fixed factors: genotype $(p<0.001)$, diet $(p<0.001)$, and enrichment $(p<0.001)$. In addition, some of the differences found among groups were because of the combination of two factors: genotype $\times$ diet $(p<0.001)$, genotype $\times$ enrichment $(p<0.001)$ or all three factors: genotype $\times$ diet $\times$ enrichment $(p<0.001)$. $B$, Representative images of IdU in the eight experimental groups. Images were acquired using a Leica Sp8 confocal microscope under $20 \times$ objective. GCL is highlighted. Scale bar, $100 \mu \mathrm{m}$. C, Survival. CldU-labeled cells 2 weeks after intraperitoneal injection. Three-way ANOVA analysis was significantly different $(p<0.001)$. These differences were because of the three individual fixed factors: genotype $(p<0.001)$, diet $(p<$ $0.001)$, and enrichment $(p<0.001)$. Some differences were also because of the combination of different factors, such as genotype $\times$ diet $(p<0.001)$, genotype $\times$ enrichment $(p<0.001)$. The combination of these three factors, genotype $\times$ diet $\times$ enrichment $(p=0.010)$, was also responsible for differences found in the experiment. $D$, Representative pictures of CldU in the eight experimental groups. Images were acquired using a Leica Sp8 confocal microscope under $20 \times$ objective. GCL is highlighted. Scale bar, $100 \mu \mathrm{m}$. E, Sox2/GFAP-positive cells in DG. Numbers of neural stem cells in DG of nontransgenic and PS1 ${ }^{\mathrm{M} 146 \mathrm{~V} /+}$ mice in SC diet - / + EE or PLX5622 diet - /+ EE. Three-way ANOVA analysis was significantly different between groups. These differences are because of the three individual factors: genotype $(p=0.000)$, diet $(p<0.001)$, and environmental enrichment $(p=0.001)$. Some differences were also because of the combination of different factors, such as genotype $\times$ diet $(p<0.0012)$ and genotype $\times$ enrichment $(p=0.0052)$. The combination of these three factors, genotype $\times$ diet $\times$ enrichment $(p<0.0011)$, was also responsible for differences found in the experiment. $\boldsymbol{F}$, Representative images of Sox2/GFAP-positive neural stem cells in the eight experimental groups shown in $\boldsymbol{E}$. Micrographs were taken using a Leica SP8 confocal microscope under $20 \times$ objective. Scale bar, $100 \mu \mathrm{m}$. G, Representative images of DCX-positive cells in the DG of the eight experimental groups shown in H. Pictures were acquired with a Leica SP8 confocal microscope, under $10 \times$ objective. Scale bar, $50 \mu \mathrm{m}$. $\boldsymbol{H}$, Total numbers of DCX-positive cells. Neuroblasts in the DG of nontransgenic and PS1 ${ }^{\mathrm{M} 146 \mathrm{~V} /+}{ }^{+}$mice in SC diet $-/+$ EE or PLX5622 diet $-1+$ EE. Three-way ANOVA showed a significant difference $(p<0.001)$. These differences are because of genotype $(p<0.001)$, diet $(p<0.001)$, and enrichment $(p<0.001)$. In addition, the combination of genotype $\times \operatorname{diet}(p=0.0029)$ and genotype $\times$ enrichment $(p=0.0196)$ was also significant. Finally, the combination of the three fixed factors was also responsible for the differences found in our groups: genotype $\times$ diet $X$ enrichment $(p<0.001)$. $\boldsymbol{I}$, Representative images of NeuN-positive mature neurons in the eight groups shown in $J$. Images were acquired using a Leica Sp8 confocal microscope under 10× objective. Scale bar, $100 \mu \mathrm{m}$. J, NeuN-positive cells in DG. Mature neurons in DG of nontransgenic and PS1 ${ }^{\mathrm{M146V} /+}$ mice in SC diet - / EE or PLX5622 diet - /+ EE. Three-way ANOVA analysis was significant $(p<0.001)$ and all differences between groups are because of genotype $(p<0.0017)$ and diet $(p=0.0010)$, whereas the other individual fixed factors and all the possible combination between factors were not significantly different ( $p>0.05$ in all cases).

$(p<0.001)$. Finally, in the case of NeuN analysis, three-way ANOVA analysis was significant $(p<0.001)$ and all differences between groups are because of genotype $(p<0.0017)$ and diet $(p=0.0010)$, whereas the other individual fixed factors and all the possible combination between factors were not significantly different ( $p>0.05$ in all cases).
Similar to our observations in the transgenic mice models, we were able to rescue all neurogenic cell populations after microglia depletion in $P S 1^{\mathrm{M} 146 \mathrm{~V} /+}$, thus, highlighting the important role of microglia in mediating the deficits in AHNPC proliferation, survival, and the different neurogenic cell populations in mice expressing FAD-linked PS1. 


\section{Microglia depletion rescues baseline anxiety in mice expressing FAD-linked PS1}

In view of studies showing that anxiety is a common clinical feature in patients with AD (Ferreira et al., 2018) and in individuals with preclinical Alzheimer's disease (Donovan et al., 2018), we chose to test the baseline anxiety of all groups of animals. Our behavioral tests, including dark/light test and marble burying test, do not inflict any stress on the animals that could impact on the process of neurogenesis. In addition, the marble burying test also provides information pertaining to compulsive behaviors that are also present in patients with AD (Nyatsanza et al., 2003). In this regard, studies have also investigated the possibility that obsessive compulsive behavior is a risk factor for AD (Dondu et al., 2015). In the dark/light test, we analyzed three independent parameters. For interpretation, we chose to represent the time in light area (Fig. $5 \mathrm{~A}$ ) that is directly related to anti-anxiety behavior (Crawley, 1985; Malmberg-Aiello et al., 2002; Bourin and Hascoët, 2003). An increase in the time spent in the light area is indicative of lower baseline anxiety. We also observed rearing behavior (Fig. 5B) as a common parameter that reports on antianxiety and exploratory behavior (Crawley, 1985; MalmbergAiello et al., 2002; Bourin and Hascoët, 2003). Finally, we examined grooming (Fig. 5D), an anxiety parameter widely described in the literature (Crawley, 1985; Malmberg-Aiello et al., 2002; Bourin and Hascoët, 2003).

Under basal conditions (SC/NO EE), mice expressing huPS1WT showed lower basal anxiety as reported by increased time spent in the light area and more rearing events compared with mice expressing the FAD-linked variants. When mice were exposed to EE and the SC diet, only the huPS1WT mice increased their time in the light area and increased their rearing behavior. However, with the PLX5622 diet, all mice, independent of genotype, increased their abilities in the test performance, spent more time in the light area, and increased rearing events (Fig. 5A-C). In the case of grooming, we observed just the opposite, as would be expected if the animals were less anxious. Thus, animals fed PLX5622 exhibited lower baseline anxiety. In the analysis of the time spent in light area, three-way ANOVA showed a significant difference $(p<0.001)$. Those differences are because of two of three independent fixed factors: genotype $(p<0.001)$ and diet $(p=0.014)$, but not to enrichment $(p=0.752)$. In addition, combination of different fixed factors also contributed to the differences observed: genotype $\times \operatorname{diet}(p<0.001)$, genotype $\times$ enrichment $(p=0.003)$, diet $\times$ enrichment $(p<0.001)$, and the combination of the three factors genotype $\times$ diet $\times$ enrichment $(p<0.001)$. Three-way ANOVA of rearing analysis showed significant differences $(p=000)$. These differences were because of the three individual factors: genotype $(p<0.001)$, diet $(p<$ $0.001)$, and enrichment $(p=0.023)$. The combination of those factors also contributed in a significantly different manner: genotype $\times \operatorname{diet}(p<0.001)$ and diet $\times$ enrichment $(p<0.001)$, while the combination of genotype $\times$ enrichment $(p=0.508)$ was not significant. The combination of the three independent factors also contributed to our final output $(p<0.001)$ in a significant manner. Finally, the three-way ANOVA of grooming behaviors showed significant differences $(p<0.001)$. Those differences were because of the effect of two individual factors: diet $(p<$ $0.001)$ and enrichment $(p=0.004)$, but not genotype $(p=$ $0.503)$. The combination of different factors did not result in significant differences in our model: genotype $\times \operatorname{diet}(p=0.807)$, genotype $\times$ enrichment $(p=0.062)$, diet $\times$ enrichment $(p=$ $0.709)$. Neither did the combination of our three independent factors: genotype $\times$ diet $\times$ enrichment $(p=0.240)$.
In the case of the marble burying test (Fig. 5G,H), we observed that under normal conditions (SC/NO EE), all genotypes performed similarly on the test. When animals were exposed to EE and the SC diet, only mice expressing huPS1WT improved their output on the test as they buried fewer marbles than in $\mathrm{SC} / \mathrm{SH}$ conditions. The mice expressing FAD-linked PS1 also improved their test performance, but to a limited degree. On the other hand, all mouse lines treated with PLX5622 and independent of EE, performed better on the test, burying fewer marbles compared with the SC groups. Thus, we conclude that PLX5622mediated microglial depletion rescues the heightened baseline anxiety observed in transgenic mice expressing FAD-linked PS1 variants. Three-way ANOVA of the marble burying test showed significant differences in the model $(p<0.001)$. Those differences were because of two of our three independent factors: diet $(p<0.001)$ and enrichment $(p<0.001)$. However genotype did not contribute to those differences $(p=0.724)$. In addition, the combination of the factors also contributed to our final output: genotype $\times \operatorname{diet}(p=0.004)$ and diet $\times$ enrichment $(p=0.002)$.

Extending these latter studies, we examined basal anxiety behaviors in $P S 1^{M 146 V /+}$ mice. As we had observed in the transgenic mice expressing PS1 variants, mice, basal anxiety in control conditions (SC/NO EE) in the $P S 1^{M 146 V /+}$ mice was similar to nontransgenic mice (Fig. 5D-F, I,J). When nontransgenic animals were subject to EE, we observed a reduction in baseline anxiety, as measured in the dark/light test where there was more time spent in light area (Fig. 5D), more rearing behavior (Fig. 5E) and less grooming behavior (Fig. $5 F$ ). In addition, we observed fewer marbles buried in the marble burying test (Fig. 5I,J). However, this EE reduction of baseline anxiety in nontransgenic animals was not observed in the $P S 1^{M 146 V /+}$ mice exposed to EE. Nonetheless, PLX5622-mediated microglia depletion reduced baseline anxiety both in nontransgenic mice and $P S 1^{M 146 V /+}$ animals, and PLX5622 treatment in combination with EE generated even lower levels of anxiety in both nontransgenic and $P S 1^{\mathrm{M} 146 \mathrm{~V} /+}$ mice (Fig. 5D-F, $I, J$ ). Three-way ANOVA of the time in light area showed that there were significant differences in our model $(p<$ $0.001)$. These differences are because of the three independent fixed factors: genotype $(p<0.001)$, diet $(p<0.001)$, and enrichment $(p<0.001)$. In addition, a combination of different fixed factors also contributed to the differences observed: genotype $\times$ diet $(p=0.012)$, genotype $\times$ enrichment $(p=0.005)$, but the combination of diet $\times$ enrichment was not significant $(p=$ $0.175)$. Finally, the combination of the three factors genotype $\times$ diet $\times$ enrichment $(p=0.001)$ was also responsible for the changes observed. Three-way ANOVA of the rearing behaviors showed significant differences $(p=000)$. These differences were because of the three individual factors: genotype $(p<0.001)$, diet $(p<0.001)$, and enrichment $(p<0.001)$. The combination of these factors also contributed in a significantly different manner: genotype $\times$ enrichment $(p=0.006)$, being more significant than the combination between genotype $\times \operatorname{diet}(p=0.180)$ and $\operatorname{diet} \times$ enrichment $(p=0.065)$. Finally the combination of the three independent factors also contributed to our final output ( $p=$ 0.001 ) in a significant manner. In the statistical analysis of grooming behaviors, we found that our model had significant differences $(p<0.001)$. These differences were because of the effect of two individual factors: $\operatorname{diet}(p<0.001)$ and enrichment ( $p=0.006)$, but not genotype $(p=0.120)$. The combination of different factors result in significant differences in our model in the case of diet $\times$ enrichment $(p=0.018)$, and there is a trend toward significance when it is considered genotype $\times$ enrichment ( $p=0.065$ ). However, neither the combination of geno- 
A $\square$ huPS1WT $\square$ huPS1DE9 $\square$ huPS1M146L

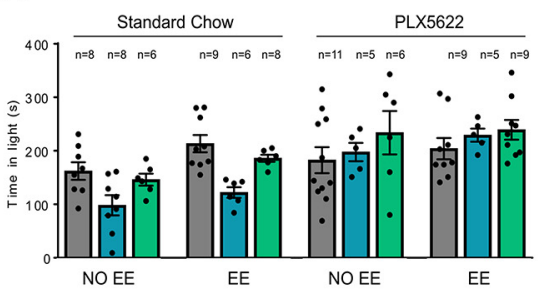

D $\square$ Non Transgenic $\square$ KI M146V

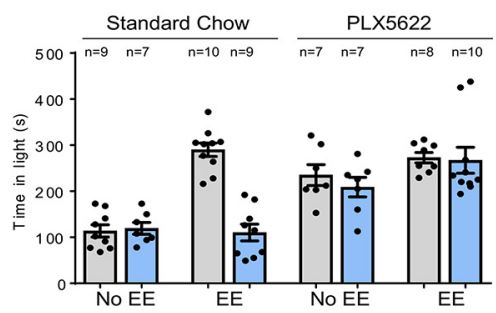

G

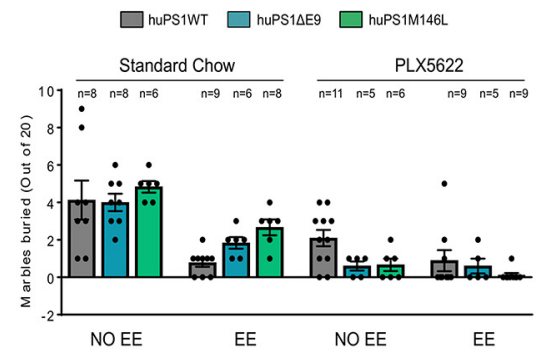

B

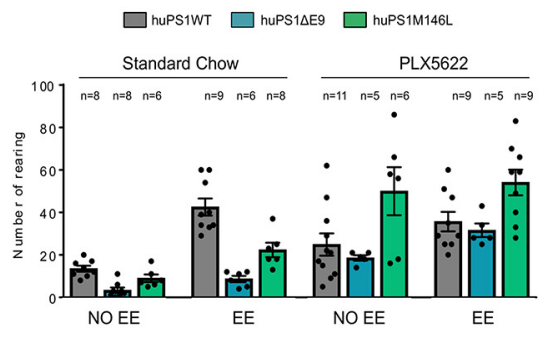

E

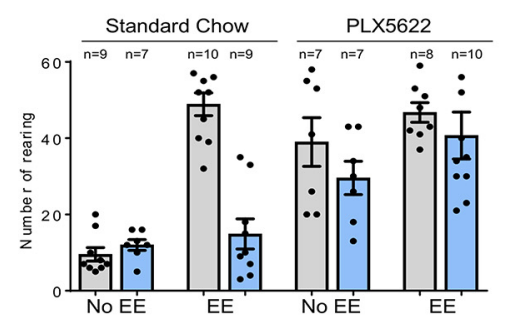

H

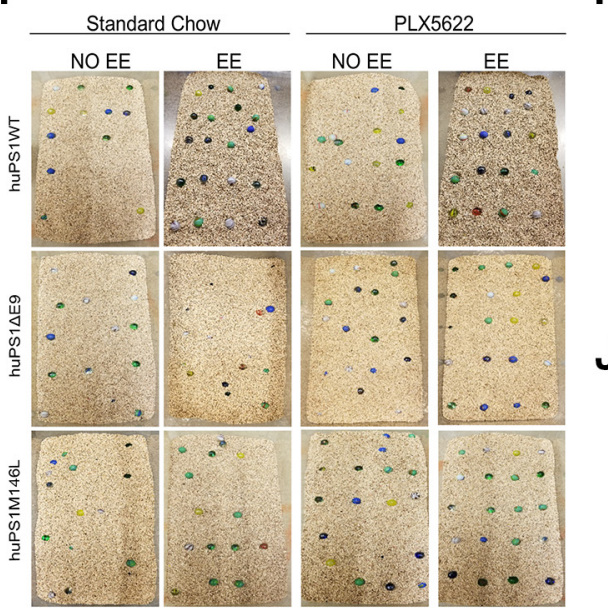

C

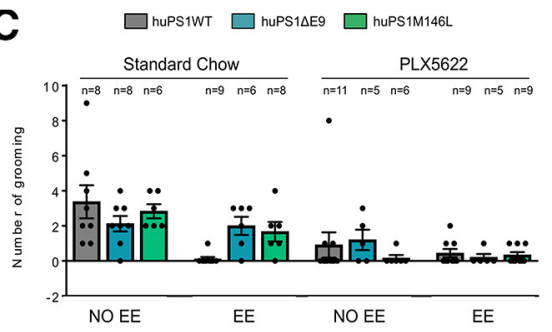

F

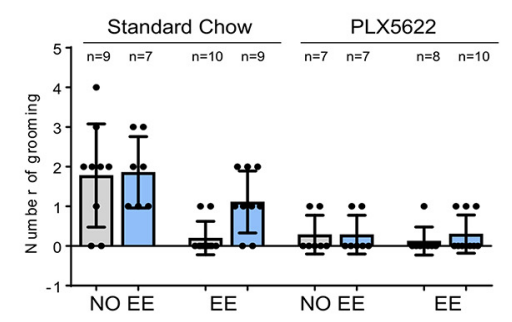

I

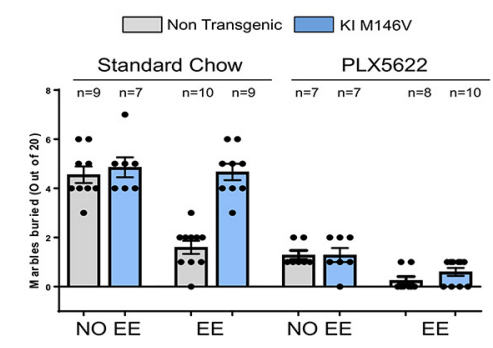

J

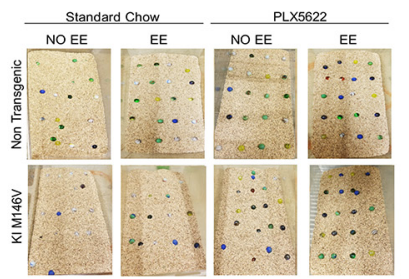

Figure 5. Analysis of basal anxiety. $A-C$, Dark/light test in transgenic mice expressing huPS1WT or FAD-linked huPS1 $\triangle E 9$ and huPS1M146L variant. $A$, Time in light area(s) of the dark/light test. Animals were tested in SC diet $-1+$ EE or PLX5622 diet $-1+$ EE. Three-way ANOVA showed a significant difference $(p<0.001)$. Those differences are because of two of three independent fixed factors: genotype $(p<0.001)$ and diet $(p=0.014)$, but not to enrichment $(p=0.752)$. In addition, combination of different fixed factors also contributed to the differences observed: genotype $X$ $\operatorname{diet}(p<0.001)$, genotype $\times$ enrichment $(p=0.003)$, diet $\times$ enrichment $(p<0.001)$, and the combination of the three factors genotype $\times$ diet $\times$ enrichment $(p<0.001)$. B, Number of rearings in the dark/light test. The number of rearings is considered an anti-anxiety behavior and is related to the exploratory activity of mice. Three-way ANOVA showed significant differences $(p=$ 000). These differences were because of the three individual factors: genotype $(p<0.001)$, diet $(p<0.001)$, and enrichment $(p=0.023)$. The combination of those factors also contributed in a significantly different manner: genotype $\times \operatorname{diet}(p<0.001)$ and diet $\times$ enrichment $(p<0.001)$, whereas the combination of genotype $\times$ enrichment $(p=0.508)$ was not significant. Finally, the combination of the three independent factors also contributed to our final output $(p<0.001)$ in a significant manner. $C$, Number of grooming events in the dark/light test. Grooming is an anxiety behavior. Three-way ANOVA showed significant differences $(p<0.001)$. Those differences were because of the effect of two individual factors: diet $(p<0.001)$ and enrichment $(p=0.004)$, but not genotype $(p=$ 0.503). The combination of different factors did not result in significant differences in our model: genotype $\times \operatorname{diet}(p=0.807)$, genotype $\times$ enrichment $(p=0.062)$, diet $\times$ enrichment $(p=0.709)$. Neither

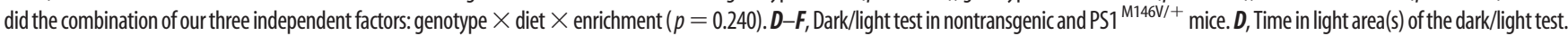
Animals were tested in SC diet $-1+$ EE or PLX5622 diet $-1+$ EE. Three-way ANOVA showed that there were significant differences in our model $(p<0.001)$. These differences are because of the three independent fixed factors: genotype $(p<0.001)$, diet $(p<0.001)$, and enrichment $(p<0.001)$. In addition, a combination of different fixed factors also contributed to the differences observed: genotype $\times$ $\operatorname{diet}(p=0.012)$, genotype $\times$ enrichment $(p=0.005)$, but the combination of diet $\times$ enrichment was not significant $(p=0.175)$. Finally, the combination of the threefactors genotype $\times$ diet $\times$ enrichment $(p=0.001)$ was also responsible for the changes observed. $E$, Number of rearings in the dark/light test. Three-way ANOVA showed significant differences $(p=000)$. These differences were because of the three individual factors: genotype $(p<0.001)$, diet $(p<0.001)$, and enrichment $(p<0.001)$. The combination of these factors also contributed in a significantly different manner: genotype $\times$ enrichment $(p=$ 0.006 ), being more significant than the combination between genotype $\times \operatorname{diet}(p=0.180)$ and diet $\times$ enrichment $(p=0.065)$. Finally the combination of the three independent factors also contributed to our final output $(p=0.001)$ in a significant manner. $F$, Number of grooming events in the dark/light test. In the statistical analysis, we found that our model had significant differences $(p<0.001)$. These differences were because of the effect of two individual factors: diet $(p<0.001)$ and enrichment $(p=0.006)$, but not genotype $(p=0.120)$. The combination of different factors result in significant differences in our model in the case of diet $\times$ enrichment $(p=0.018)$, and there is a trend toward significance when itis considered genotype $\times$ enrichment $(p=0.065)$. However, neither the combination of genotype $\times$ $\operatorname{diet}(p=0.274)$ nore the combination of our three independent factors: genotype $\times \operatorname{diet} \times$ enrichment $(p=0.165)$ were significant. $\boldsymbol{G}, \boldsymbol{H}$, Marble burying test in transgenic mice expressing huPS1WT or FAD-linked huPS1 $\triangle$ E9 and huPS1M146L variant. G, Graphical results of the marble burying test in the 12 experimental groups. Three-way ANOVA showed significant differences in the model $(p<0.001)$. Those differences were because of two of our three independent factors: diet $(p<0.001)$ and enrichment $(p<0.001)$. However genotype did not contribute to those differences $(p=0.724)$. In addition, the combination of the factors also contributed to our final output: genotype $\times \operatorname{diet}(p=0.004)$ and diet $\times$ enrichment $(p=0.002)$. $\boldsymbol{H}$, Representative image of the marble burying test at the end of the experiment. $I, J$, Marble burying test in nontransgenic and PS $1^{\mathrm{M} 146 \mathrm{~V} /+}$ mice. $I$, Graphical results of the marbles buried (of 20) in the eight experimental groups. Statistical analysis showed significant differences in the model $(p<0.001)$. These differences were because of the three independent factors: genotype $(p<0.001)$, diet $(p<0.001)$, and enrichment $(p<0.001)$. In addition, the combination of the factors also contributed to our final output: genotype $\times \operatorname{diet}(p<0.001)$, genotype $\times$ enrichment $(p<0.001)$, and there is a trend toward significance in diet $\times$ enrichment $(p=0.093)$. The combination of the three different fixed factors also contribute to the differences observed in our model $(p=0.003) . J$, Representative image of the marble burying test at the end of the experiment. 
A

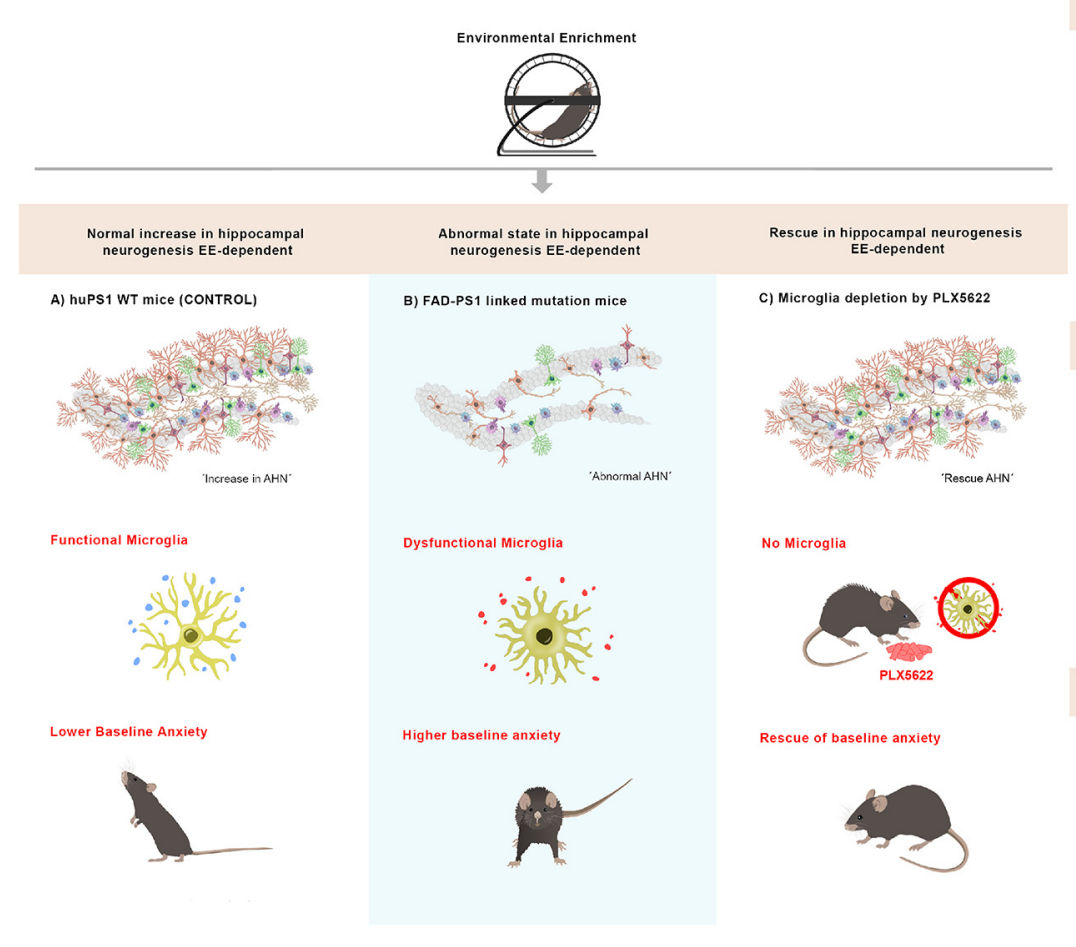

B
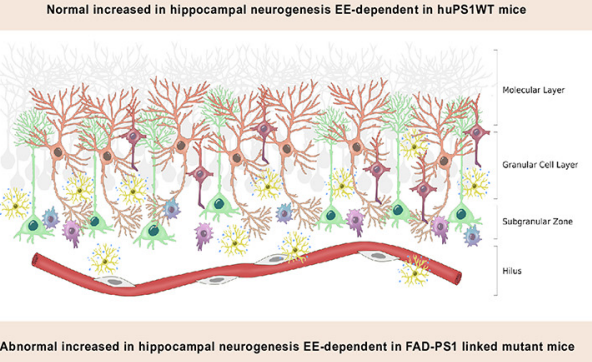

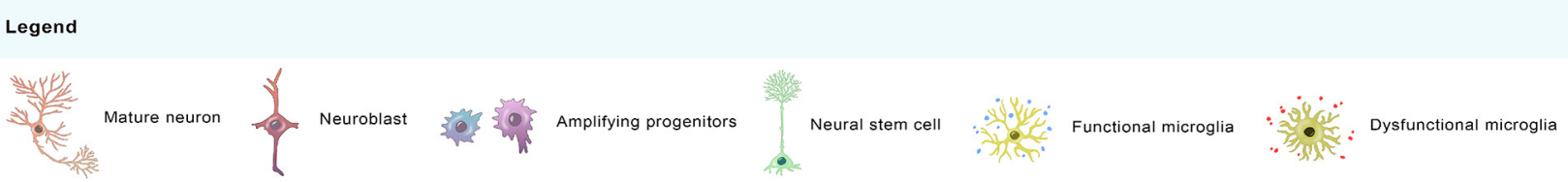

Figure 6. Graphical abstract: summary of the results in of our studies. A, Global scheme of the results we obtained after EE in the three main conditions (control mice, huPS1WT mice under EE and SC diet; FAD-PS1-linked mutant mice under EE and SC diet; FAD-PS1-linked mutant mice under EE and PLX5622 diet). Hippocampal neurogenesis, the role of microglia, and the baseline anxiety are depicted. $\boldsymbol{B}$, Neurogenic cell details in the aforementioned conditions of our work. The cell types in the GCL, SGZ in the DG, and hilus are shown in the legend.

type $\times$ diet $(p=0.274)$ nor the combination of our three independent factors: genotype $\times \operatorname{diet} \times$ enrichment $(p=0.165)$ were significant. Finally, three-way ANOVA of marble burying test showed significant differences in the model $(p<0.001)$. Those differences were because of two of our three independent factors: $\operatorname{diet}(p<0.001)$ and enrichment $(p<0.001)$. However genotype did not contribute to those differences $(p=0.724)$. In addition, the combination of the factors also contributed to our final output: genotype $\times \operatorname{diet}(p=0.004)$ and diet $\times$ enrichment $(p=0.002)$.

\section{Discussion}

In an effort to establish that microglia in mice expressing mutant PS1 play a critical role in the regulation of proliferation and differentiation of AHNPCs, we chose a strategy in which microglia were ablated with a highly selective CSF1R antagonist and now offer several important insights. First, we have confirmed our previous findings that expression of mouse $\mathrm{PrP}$ promoter-driven transgenes encoding FAD-linked PS1 variants impairs EEinduced AHNPC proliferation, survival, and neuronal differentiation, as well as the levels of neural stem cells, amplifying progenitors and maturation of neuroblasts compared with mice expressing huPS1WT. Supporting these studies, the proliferation and survival of AHNPCs in $P S 1^{M 146 V /+}$ mice is significantly com- promised compared with nontransgenic littermates. Similar results were obtained for neural stem cells and neuroblasts in this model. Second, we now report that transgenic mice expressing FAD-PS1 linked variants and $P S 1^{M 146 V /+}$ mice exhibit heightened baseline anxiety compared with mice expressing human huPS1WT or nontransgenic mice, respectively. Following EE, mice expressing huPS1WT or nontransgenic mice showed an improvement in baseline anxiety, but baseline anxiety still persisted in mice expressing the FAD-linked PS1 variants. Third, and most importantly, microglial depletion in transgenic mice expressing the PS1 variants or $P S 1^{M 146 V /+}$ mice led to a complete restoration of EE-mediated AHNPC phenotypes and behavioral impairments to levels observed in mice expressing either human wild-type PS1 or nontransgenic mice, respectively. Finally, we observed that EE led to an elevation in the total numbers of mature neurons in the DG in all three transgenic lines and $P S 1^{M 146 V /+}$ mice after PLX5622-mediated depletion of microglia. A diagrammatic overview of these results is presented in the graphical abstract (Fig. 6A, $B$ ).

We now report, for the first time, that a CSF1R antagonist improves baseline anxiety that is observed in mice expressing transgene-encoded FAD-linked PS1 variants and in $P S 1^{M 146 V /+}$ mice. These findings suggest that microglia are responsible for 
the impairments in emotional behaviors that are directly correlated with hippocampal neurogenesis. Although there are no apparent differences in the three transgenic lines in terms of neurogenic cell fates under basal conditions (SC/ NO EE), we speculate that microglia in these animals are functional and regulate the fate of AHNPCs in a similar manner as previously reported (Sierra et al., 2010; Gemma and Bachstetter, 2013). However, in mice expressing the PS1 variants and subject to EE, microglial cells expressing FAD-PS1 variants perturb AHNPC proliferation, maturation, and survival, presumably via the secretion of soluble factors into the microenvironment that are inhibitory for these processes (Fig. 6). Supporting this proposal, microglial depletion, and hence, the loss of inhibitory factors, leads to the restoration in AHNPC proliferation and neurogenic phenotypes in mice expressing PS1 variants to the levels seen in wild-type mice. Unresolved are the nature of the factors secreted by microglia expressing wild-type of mutant PS1 in vivo after EE and the signaling pathways that are responsible for the observed suppression of AHNPC phenotypes but future transcriptomic and proteomic studies of purified microglia from transgenic mice expressing mutant PS1 or PS1 ${ }^{M 146 V /+}$ mice that coexpress microglial promoter-driven GFP or EGFP-tagged ribosome protein Rpl10a will be essential and are investigations currently ongoing.

One caveat of the studies reported herein is that the mice are very young and we do not have any information as to the impact of PLX5622-mediated microglial depletion on enrichmentmediated neurogenesis in older animals. The only information pertaining to microglial depletion in older animals is comprised of studies that have examined the impact of PLX5622-mediated microglial depletion on amyloid plaque burden and cognition in mouse models of $\mathrm{A} \beta$ amyloidosis. For example, Dagher et al. (2015) showed a positive effect on cognitive behaviors using the PLX5622 diet in 15-month-old 3xTg-AD mice, but with no effect on plaque burden or plaque size. Subsequently, Unger et al. (2018) reported that in 12-month-old APP-PS1 transgenic mice that also have significant levels of amyloid burden and fed with PLX5622 diet, there was neither an impact on plaque burden, nor cognition. The differences in behavioral outcomes reported in the two mouse models could easily be explained by contrasting experimental protocols in each study, and influences of housing conditions, animal gender, and age and feeding (Gemma and Bachstetter, 2013). Finally, it is important to note that the bulk of the studies on microglia depletion on pathology and behavior have been performed in mice expressing FAD-linked APP and/or PS1 variants and it is unclear whether behavioral or pathological parameters in mice expressing genes that are linked to late-onset $\mathrm{AD}$, including ApoE4, TREM2, variants, etc., or mice expressing mutant tau variants will also be modified. In this regard, we are unaware of reports that have assessed the role of microglia in the modulation of tau pathology.

In summary, we have demonstrated that microglia in mice expressing FAD-linked PS1 variants have deleterious effects on hippocampal neurogenesis and that these deficits are correlated with impairments in emotional function. In this regard, to the widely held view that FAD-linked PS1 variants cause disease by elevating the ratio of $\mathrm{A} \beta 42$ to $\mathrm{A} \beta 40$ that drives nucleation/oligomer formation, amyloid deposition, and synaptic dysfunction, which leads to cognitive deficits, our present findings offer the tantalizing suggestion that the memory deficits and cognitive decline in patients that harbor PSEN1 gene variants is a reflection of impairments in the self-renewal, survival, and differentiation of AHNPCs. That we can restore these deficits by microglial depletion now offer new opportunities to identify and target those factors driving microglial (dys)function that could be therapeutically efficacious for patients with $\mathrm{AD}$.

\section{References}

Angoa-Perez M, Kane MJ, Briggs DI, Francescutti DM, Kuhn DM (2013) Marble burying and nestlet shredding as tests of repetitive, compulsivelike behaviors in mice. J Vis Exp 82:e50978.

Bloom GS (2014) Amyloid-beta and tau: the trigger and bullet in Alzheimer disease pathogenesis. JAMA Neurol 71:505-508.

Bonds JA, Kuttner-Hirshler Y, Bartolotti N, Tobin MK, Pizzi M, Marr R, Lazarov O (2015) Presenilin-1 dependent neurogenesis regulates hippocampal learning and memory. PLoS One 10:e0131266.

Bourin M, Hascoët M (2003) The mouse light/dark box test. Eur J Pharmacol 463:55-65.

Cai Z, Hussain MD, Yan LJ (2014) Microglia, neuroinflammation, and beta-amyloid protein in Alzheimer's disease. Int J Neurosci 124:307-321.

Choi SH, Veeraraghavalu K, Lazarov O, Marler S, Ransohoff RM, Ramirez JM, Sisodia SS (2008) Non-cell-autonomous effects of presenilin 1 variants on enrichment-mediated hippocampal progenitor cell proliferation and differentiation. Neuron 59:568-580.

Christian KM, Song H, Ming GL (2014) Functions and dysfunctions of adult hippocampal neurogenesis. Annu Rev Neurosci 37:243-262.

Crawley JN (1985) Exploratory behavior models of anxiety in mice. Neurosci Biobehav Rev 9:37-44.

Dagher NN, Najafi AR, Kayala KM, Elmore MR, White TE, Medeiros R, West BL, Green KN (2015) Colony-stimulating factor 1 receptor inhibition prevents microglial plaque association and improves cognition in 3xTg-AD mice. J Neuroinflammation 12:139.

De Strooper B (2003) Aph-1, pen-2, and nicastrin with presenilin generate an active gamma-secretase complex. Neuron 38:9-12.

Deng W, Aimone JB, Gage FH (2010) New neurons and new memories: how does adult hippocampal neurogenesis affect learning and memory? Nat Rev Neurosci 11:339-350.

Dondu A, Sevincoka L, Akyol A, Tataroglu C (2015) Is obsessivecompulsive symptomatology a risk factor for Alzheimer-type dementia? Psychiatry Res 225:381-386.

Donovan NJ, Locascio JJ, Marshall GA, Gatchel J, Hanseeuw BJ, Rentz DM, Johnson KA, Sperling RA; Harvard Aging Brain Study (2018) Longitudinal association of amyloid beta and anxious-depressive symptoms in cognitively normal older adults. Am J Psychiatry 175:530-537.

Elder GA, Tezapsidis N, Carter J, Shioi J, Bouras C, Li HC, Johnston JM, Efthimiopoulos S, Friedrich VL Jr, Robakis NK (1996) Identification and neuron specific expression of the $\mathrm{S} 182 /$ presenilin I protein in human and rodent brains. J Neurosci Res 45:308-320.

Erblich B, Zhu L, Etgen AM, Dobrenis K, Pollard JW (2011) Absence of colony stimulation factor-1 receptor results in loss of microglia, disrupted brain development and olfactory deficits. PLoS One 6:e26317.

Feng R, Rampon C, Tang YP, Shrom D, Jin J, Kyin M, Sopher B, Miller MW, Ware CB, Martin GM, Kim SH, Langdon RB, Sisodia SS, Tsien JZ (2001) Deficient neurogenesis in forebrain-specific presenilin-1 knockout mice is associated with reduced clearance of hippocampal memory traces. Neuron 32:911-926.

Ferreira MDC, Abreu MJ, Machado C, Santos B, Machado Á, Costa AS (2018) Neuropsychiatric profile in early versus late onset Alzheimer's disease. Am J Alzheimers Dis Other Demen 33:93-99.

Gadadhar A, Marr R, Lazarov O (2011) Presenilin-1 regulates neural progenitor cell differentiation in the adult brain. J Neurosci 31:2615-2623.

Gemma C, Bachstetter AD (2013) The role of microglia in adult hippocampal neurogenesis. Front Cell Neurosci 7:229.

Guo Q, Fu W, Sopher BL, Miller MW, Ware CB, Martin GM, Mattson MP (1999) Increased vulnerability of hippocampal neurons to excitotoxic necrosis in presenilin-1 mutant knock-in mice. Nat Med 5:101-106.

Hill AS, Sahay A, Hen R (2015) Increasing adult hippocampal neurogenesis is sufficient to reduce anxiety and depression-like behaviors. Neuropsychopharmacology 40:2368-2378.

Kim KK, Adelstein RS, Kawamoto S (2009) Identification of neuronal nuclei $(\mathrm{NeuN})$ as fox-3, a new member of the fox-1 gene family of splicing factors. J Biol Chem 284:31052-31061.

Lah JJ, Heilman CJ, Nash NR, Rees HD, Yi H, Counts SE, Levey AI (1997) Light and electron microscopic localization of presenilin-1 in primate brain. J Neurosci 17:1971-1980.

Lee HJ, Jung KM, Huang YZ, Bennett LB, Lee JS, Mei L, Kim TW (2002) 
Presenilin-dependent gamma-secretase-like intramembrane cleavage of ErbB4. J Biol Chem 277:6318-6323.

Lee MK, Borchelt DR, Kim G, Thinakaran G, Slunt HH, Ratovitski T, Martin LJ, Kittur A, Gandy S, Levey AI, Jenkins N, Copeland N, Price DL, Sisodia SS (1997) Hyperaccumulation of FAD-linked presenilin 1 variants in vivo. Nat Med 3:756-760.

Llorens-Martín M, Jurado-Arjona J, Avila J, Hernández F (2015) Novel connection between newborn granule neurons and the hippocampal CA2 field. Exp Neurol 263:285-292.

Malmberg-Aiello P, Ipponi A, Bartolini A, Schunack W (2002) Mouse light/ dark box test reveals anxiogenic-like effects by activation of histamine $\mathrm{H} 1$ receptors. Pharmacol Biochem Behav 71:313-318.

Matsumori Y, Hong SM, Fan Y, Kayama T, Hsu CY, Weinstein PR, Liu J (2006) Enriched environment and spatial learning enhance hippocampal neurogenesis and salvages ischemic penumbra after focal cerebral ischemia. Neurobiol Dis 22:187-198.

Meda L, Cassatella MA, Szendrei GI, Otvos L Jr, Baron P, Villalba M, Ferrari D, Rossi F (1995) Activation of microglial cells by beta-amyloid protein and interferon-gamma. Nature 374:647-650.

Mendez-David I, David DJ, Darcet F, Wu MV, Kerdine-Römer S, Gardier AM, Hen R (2014) Rapid anxiolytic effects of a 5-HT(4) receptor agonist are mediated by a neurogenesis-independent mechanism. Neuropsychopharmacology 39:1366-1378.

Mohammad H, Marchisella F, Ortega-Martinez S, Hollos P, Eerola K, Komulainen E, Kulesskaya N, Freemantle E, Fagerholm V, Savontous E, Rauvala H, Peterson BD, van Praag H, Coffey ET (2018) JNK1 controls adult hippocampal neurogenesis and imposes cell-autonomous control of anxiety behaviour from the neurogenic niche. Mol Psychiatry 23:487.

Neumann H, Kotter MR, Franklin RJ (2009) Debris clearance by microglia: an essential link between degeneration and regeneration. Brain 132:288-295.

Nyatsanza S, Shetty T, Gregory C, Lough S, Dawson K, Hodges JR (2003) A study of stereotypic behaviours in Alzheimer's disease and frontal and temporal variant frontotemporal dementia. J Neurol Neurosurg Psychiatry $74: 1398-1402$.

Ortega-Martínez S (2015) A new perspective on the role of the CREB family of transcription factors in memory consolidation via adult hippocampal neurogenesis. Front Mol Neurosci 8:46.

Ortega-Martínez S, Trejo JL (2015) The postnatal origin of adult neural stem cells and the effects of glucocorticoids on their genesis. Behav Brain Res 279:166-176.

Patel S, Player MR (2009) Colony-stimulating factor-1 receptor inhibitors for the treatment of cancer and inflammatory disease. Curr Top Med Chem 9:599-610.

Plümpe T, Ehninger D, Steiner B, Klempin F, Jessberger S, Brandt M, Römer B, Rodriguez GR, Kronenberg G, Kempermann G (2006) Variability of doublecortin-associated dendrite maturation in adult hippocampal neu- rogenesis is independent of the regulation of precursor cell proliferation. BMC Neurosci 7:77.

Price DL, Sisodia SS (1998) Mutant genes in familial Alzheimer's disease and transgenic models. Annu Rev Neurosci 21:479-505.

Quesseveur G, David DJ, Gaillard MC, Pla P, Wu MV, Nguyen HT, Nicolas V, Auregan G, David I, Dranovsky A, Hantraye P, Hen R, Gardier AM, Déglon N, Guiard BP (2013) BDNF overexpression in mouse hippocampal astrocytes promotes local neurogenesis and elicits anxiolyticlike activities. Transl Psychiatry 3:e253.

Ravichandran KS (2003) "Recruitment signals" from apoptotic cells: invitation to a quiet meal. Cell 113:817-820.

Revest JM, Dupret D, Koehl M, Funk-Reiter C, Grosjean N, Piazza PV, Abrous DN (2009) Adult hippocampal neurogenesis is involved in anxiety-related behaviors. Mol Psychiatry 14:959-967.

Sierra A, Encinas JM, Deudero JJ, Chancey JH, Enikolopov G, OverstreetWadiche LS, Tsirka SE, Maletic-Savatic M (2010) Microglia shape adult hippocampal neurogenesis through apoptosis-coupled phagocytosis. Cell Stem Cell 7:483-495.

Squire LR (1992) Memory and the hippocampus: a synthesis from findings with rats, monkeys, and humans. Psychol Rev 99:195-231.

Sztainberg Y, Chen A (2010) An environmental enrichment model for mice. Nat Protoc 5:1535-1539.

Thinakaran G, Borchelt DR, Lee MK, Slunt HH, Spitzer L, Kim G, Ratovitsky T, Davenport F, Nordstedt C, Seeger M, Hardy J, Levey AI, Gandy SE, Jenkins NA, Copeland NG, Price DL, Sisodia SS (1996) Endoproteolysis of presenilin 1 and accumulation of processed derivatives in vivo. Neuron $17: 181-190$

Thinakaran G, Harris CL, Ratovitski T, Davenport F, Slunt HH, Price DL, Borchelt DR, Sisodia SS (1997) Evidence that levels of presenilins (PS1 and PS2) are coordinately regulated by competition for limiting cellular factors. J Biol Chem 272:28415-28422.

Torres-Lista V, López-Pousa S, Giménez-Llort L (2015) Marble-burying is enhanced in $3 \mathrm{xTg}-\mathrm{AD}$ mice, can be reversed by risperidone and it is modulable by handling. Behav Processes 116:69-74.

Unger MS, Schernthaner P, Marschallinger J, Mrowetz H, Aigner L (2018) Microglia prevent peripheral immune cell invasion and promote an antiinflammatory environment in the brain of APP-PS1 transgenic mice. J Neuroinflammation 15:274.

Veeraraghavalu K, Sisodia SS (2013) Mutant presenilin 1 expression in excitatory neurons impairs enrichment-mediated phenotypes of adult hippocampal progenitor cells. Proc Natl Acad Sci U S A 110:9148-9153.

Veeraraghavalu K, Choi SH, Zhang X, Sisodia SS (2013) Endogenous expression of FAD-linked PS1 impairs proliferation, neuronal differentiation and survival of adult hippocampal progenitors. Mol Neurodegener $8: 41$.

Weitz TM, Town T (2012) Microglia in Alzheimer's disease: it's all about context. Int J Alzheimers Dis 2012:314185. 